\title{
Numerical Approaches to Compute Spectra of Non-Self Adjoint Operators and Quadratic Pencils
}

\author{
Fatima Aboud ${ }^{1}$, François Jauberteau ${ }^{2, *}$, Guy Moebs ${ }^{2}$ \\ and Didier Robert ${ }^{2}$ \\ ${ }^{1}$ Mathematics Department, College of Science, University of Diyala, Baquba, Iraq \\ ${ }^{2}$ Laboratoire de Mathématiques Jean Leray, CNRS-UMR 6629, Université de Nantes, \\ France.
}

Received December 26, 2018; Accepted June 28, 2019;

Published online February 28, 2020

\begin{abstract}
In this article we are interested in the numerical computation of spectra of non-self adjoint quadratic operators. This leads to solve nonlinear eigenvalue problems. We begin with a review of theoretical results for the spectra of quadratic operators, especially for the Schrödinger pencils. Then we present the numerical methods developed to compute the spectra : spectral methods and finite difference discretization, in infinite or in bounded domains. The numerical results obtained are analyzed and compared with the theoretical results. The main difficulty here is that we have to compute eigenvalues of strongly non-self-adjoint operators which are very unstable.
\end{abstract}

AMS subject classifications: 47A75, 47F05, 65N06, 65N25, 65N35

Key words: Nonlinear eigenvalue problems, spectra, pseudospectra, finite difference methods, Galerkin spectral method, Hermite functions.

\section{Introduction}

We are interested here in equations like $L(\lambda) u=0$ where $L(\lambda)$ is a linear operator on some linear space $\mathcal{E}$, depending on a complex parameter $\lambda$. When $L(\lambda)=L_{0}-\lambda \mathbb{I}$, this is the usual eigenvalue problem : find $\lambda \in \mathbb{C}$ and $u \in \mathcal{E}, u \neq 0$ such that $L(\lambda) u=0$. In many applications, in particular for dissipative problems in mechanics, it is necessary to consider more general dependence in the complex parameter $\lambda$. A particular interesting case is a quadratic family of operators: $L(\lambda)=\lambda^{2} L_{2}+\lambda L_{1}+L_{0}$, where $L_{2}, L_{1}$ and $L_{0}$ are linear operators in $\mathcal{E}$. We shall say that $L(\lambda)$ is a quadratic pencil.

\footnotetext{
*Corresponding author. Email addresses: Fatima.Aboud@sciences.uodiyala.edu.iq (F. Aboud), francois.jauberteau@univ-nantes.fr (F. Jauberteau), guy.moebs@univ-nantes.fr (G. Moebs), didier.robert@univ-nantes.fr (D. Robert)
} 
Let us consider the second order differential equation :

$$
L_{2}\left(\frac{d^{2} u}{d t^{2}}\right)+L_{1}\left(\frac{d u}{d t}\right)+L_{0}(u)=0
$$

Eq. (1.1) is a model in mechanics for small oscillations of a continuum system in the presence of an impedance force [26]. Now by looking for stationary solutions of (1.1), $u(t)=u_{0} \mathrm{e}^{\lambda t}$, we have the following equation:

$$
\left(\lambda^{2} L_{2}+\lambda L_{1}+L_{0}\right) u_{0}=0 .
$$

So Eq. (1.2) is a non-linear eigenvalue problem in the spectral parameter $\lambda \in \mathbb{C}$. We say that $\lambda$ is a non-linear eigenvalue if there exists $u_{0} \neq 0$ satisfying (1.2).

The operator $L_{1}$ represents a damping term as we see in the following simple example. Let us consider the perturbed wave equation (see [5]) :

$$
\frac{\partial^{2}}{\partial t^{2}} u-\frac{\partial^{2}}{\partial^{2} x} u-2 a \frac{\partial}{\partial t} u=0,
$$

where $t \in \mathbb{R}$ and $x \in \mathbb{T}:=\mathbb{R} / 2 \pi \mathbb{Z}$. Here we have $L_{2}=\mathbb{I}_{\mathcal{E}}$ (identity operator), $L_{1}=-2 a$ and $L_{0}=-\partial^{2} / \partial x^{2}$. The damping term $a<0$ is here constant. So we have to solve (1.3) with periodical boundary conditions. The stationary problem is reduced to the equation :

$$
\lambda^{2}+k^{2}-2 a \lambda=0, \quad k \in \mathbb{Z}
$$

Then we have for $k^{2} \geq a^{2}$ the damped solutions of (1.3):

$$
u_{k}(t, x)=\exp \left(\left(a+i \sqrt{k^{2}-a^{2}}\right) t+i k x\right) .
$$

When $a$ is a function of $x$ we have no explicit formula so we need numerical approximations to compute the damping modes. It is the main goal of this work.

Such generalized eigenvalue problems have appeared in a completely different way. The question was to decide if a class of P.D.E with analytic coefficients preserves or not the analyticity property. To be more explicit, let us consider a P.D.E : $P u=f$. Assume that $f$ is analytic in some open set $\Omega$, is-it true that $u$ is analytic in $\Omega$ ? This is true for elliptic operators. For some example, this question can be reduced to the following (see [23] for more details) : does there exist $\lambda \in \mathbb{C}, 0 \neq u \in \mathcal{S}(\mathbb{R})$ such that

$$
\left(-\frac{d^{2}}{d x^{2}}+\left(x^{2}-\lambda\right)^{2}\right) u=0 ?
$$

Existence of non null solutions for (1.2) and (1.4) is a non trivial problem. For (1.4) it was solved in [33] where it is proved that the generalized eigenfunctions span the Hilbert space $L^{2}(\mathbb{R})$. 
On the other side we can prove that the equation :

$$
\left(-\frac{d^{2}}{d x^{2}}+(x-\lambda)^{2}\right) u=0
$$

has only the trivial solution $u \equiv 0$ in $L^{2}(\mathbb{R}), \forall \lambda \in \mathbb{C}$.

Our aim in this work is to present several numerical approaches concerning this kind of non-linear eigenvalue problems. For simplicity we only consider quadratic pencil such that $L_{0}=\mathbb{I}$. We can reduce to this case if $L_{0}$ or $L_{2}$ are invertible in the linear space $\mathcal{E}$. To every quadratic pencil $L(\lambda)$ we can associate a linear operator $\mathcal{A}_{L}$ in $\mathcal{E} \times \mathcal{E}$ such that $\lambda$ is a non-linear eigenvalue for $L$ if and only if $\lambda$ is a usual eigenvalue for $\mathcal{A}_{L}$. The operator $\mathcal{A}_{L}$ is called a linearization of $L(\lambda)$. It is easy to see that we can choose :

$$
\mathcal{A}_{L}=\left(\begin{array}{cc}
0 & \text { II } \\
-L_{0} & -L_{1}
\end{array}\right)
$$

So non-linear eigenvalue problems (for polynomial operator pencils) can be reduced to usual eigenvalue problems but it is useful to take care of their particular structure. There exists infinitely many linearizations.

We are mainly interested here in the case called Schrödinger pencils :

$$
L_{V, a}(\lambda)=-\triangle+V-2 a \lambda+\lambda^{2},
$$

where $V$ and $a$ are real smooth functions from $\mathbb{R}^{m}$ into $\mathbb{R}$ (see Section 2.2 for more details). The main questions we want to discuss is the localization of the eigenvalues of $L_{V, a}$ in the complex plane $\mathbb{C}$.

In Section 2 we shall recall some known theoretical results. We shall represent some accurate theoretical results on the location of the eigenvalues have been obtained for 1D pencils $L_{V, a}$ but in the multidimensional case very few results are known on the eigenvalues of $L_{V, a}$ when $a$ is of the same order of $\sqrt{V}$.

In Section 3 we discuss several numerical approaches for the computation of the spectra of quadratic pencil, in infinite and in bounded domain. Using spectral methods or finite difference discretization to approximate the operators $L_{0}, L_{1}$ and $L_{2}$, we obtain a nonlinear eigenvalue problem. Then, after linearization we obtain an eigenvalue problem to solve. The numerical results obtained are analyzed and comparisons with theoretical results are done. The main difficulty is that we have to compute eigenvalues of strongly non self-adjoint operators, which leads after discretization to strongly unstable nonlinear eigenvalue problems. Finally, in Section 4 we give conclusions and future works.

\section{A review of results obtained by functional analysis methods}

In this paper our main goal is to locate, in the complex plane, the generalized spectrum of pencils of differential operators. These generalized eigenvalues are very unstable and 
the theorem of existence of eigenvalues may be difficult to prove. Moreover for numerical results it is difficult to make the difference between the eigenvalues and pseudoeigenvalues.

Of course there exists a huge number of papers concerning computations of eigenvalues. Here we only notice some references directly connected with our paper.

For estimation of the spectra of linear differential operators we can refer to $[3,7,8,11,12$, $22,28,32]$. For numerical methods to solve nonlinear eigenvalue problems (finite dimensional case) and for linearization of such nonlinear eigenvalue problems we can refer to $[5,6,9,19,21,31,36,39]$.

\subsection{Quadratic family operators}

For more details on the results presented in this section we refer to the book [30].

Let us consider the quadratic family of operators $L(\lambda)=L_{0}+\lambda L_{1}+\lambda^{2}$ where $L_{0}, L_{1}$ are operators in an Hilbert space $\mathcal{H}$. If $\mathcal{H}$ if of dimension $N<+\infty$ the eigenvalues are the solutions of the polynomial equation $\operatorname{det}(L(\lambda))=0$. When $N$ is large this could be a difficult problem at least for numerical computations. In applications involving PDE, $\mathcal{H}$ is a $L^{2}$ space or a Sobolev space, which is infinite dimensional and there is no explicit equation for the generalized eigenvalues. Moreover, as we shall see later, the non linear eigenvalue problem is equivalent to a linear eigenvalue problem which, in general, is non self-adjoint hence unstable.

$L_{0}$ is assumed to be self-adjoint, positive, with a domain $D\left(L_{0}\right)$ and $L_{1}$ is $\sqrt{L_{0}}$-bounded. Moreover $L_{0}^{-1 / 2}$ is in a Schatten class $\mathcal{C}^{p}(\mathcal{H})^{\dagger}$, for some real $p>0$. We have the following results.

Theorem 2.1. $L(\lambda)$ is a family of closed operators in $\mathcal{H} . \lambda \mapsto L^{-1}(\lambda)$ is meromorphic in the complex plane. The poles $\lambda_{j}$ of $L^{-1}(\lambda)$, with multiplicity $m_{j}$, coincide with the eigenvalues with the same multiplicities, of the matrix operator $\mathcal{A}_{L}$ in the Hilbert space $\mathcal{H} \times D\left(L_{0}^{1 / 2}\right)$, with domain $D\left(\mathcal{A}_{L}\right)=D\left(L_{0}\right) \times D\left(L_{0}^{1 / 2}\right)$ where

$$
\mathcal{A}_{L}=\left(\begin{array}{cc}
0 & \mathbb{I} \\
-L_{0} & -L_{1}
\end{array}\right) \text {. }
$$

Assuming that $V(x) \geq C|x|^{2 m}$ and $|a(x)| \leq C \sqrt{V(x)}, C>0$, then the Schrödinger pencil $L_{V, a}(\lambda)$ satisfies the above theorem for $p>\frac{d(m+1)}{2 m}$.

If $L_{0}$ is positive and non degenerate we have the symmetric linearization :

$$
\mathcal{A}_{S L}=\left(\begin{array}{cc}
0 & \sqrt{L_{0}} \\
-\sqrt{L_{0}} & -L_{1}
\end{array}\right) .
$$

Let us denote $\mathrm{Sp}[L]$ the eigenvalues of $\mathcal{A}_{L}$ (which coincide with the poles of $L^{-1}(z)$ ).

\footnotetext{
${ }^{\dagger}$ Recall that a compact operator $A$ in an Hilbert space is in the Schatten class $\mathcal{C}^{p}$ if the series $s_{j}(A)$ of the eigenvalues of $\sqrt{A^{*} A}$ satisfies $\sum s_{j}(A)^{p}<+\infty$
} 
Remark 2.1. It may happens that $\mathrm{Sp}[L]$ is empty (for example if $L(\lambda)=-\frac{d^{2}}{d x^{2}}+(x-\lambda)^{2}$ ).

Let us remark that if $L_{1}=0$ then $\lambda \in \operatorname{Sp}[L]$ if and only if $-\lambda^{2}$ is in the spectrum of $L_{0}$. So if $L_{0}$ has a point spectrum then $\mathrm{Sp}[L]$ is a subset of the imaginary axis. We shall see now that when $L_{1}$ is strictly smaller than $\sqrt{L}_{0}$ then the eigenvalues are asymptotically close to the imaginary axis and the generalized eigenvectors is a dense set in the Hilbert space. When $L_{1}$ has the same order as $\sqrt{L_{0}}$ (i.e. $c_{1} \sqrt{L_{0}} \leq L_{1} \leq c_{2} \sqrt{L_{0}}$ with $c_{1}$ and $c_{2}$ positive constants) it may happens that there is no eigenvector at all for $L(\lambda)$.

If $\lambda_{0} \in \operatorname{Sp}[L]$ we denote by $\mathcal{E}_{L}\left(\lambda_{0}\right)$ the linear space of the solutions $\left\{u_{0}, u_{1}, \cdots, u_{k}, \cdots\right\}$ of the equations :

$$
\begin{aligned}
& L\left(\lambda_{0}\right) u_{0}=0, \quad L(\lambda) u_{1}+L^{\prime}\left(\lambda_{0}\right) u=0, \\
& L\left(\lambda_{0}\right) u_{k+2}+L^{\prime}\left(\lambda_{0}\right) u_{k+1}+\frac{1}{2} L^{\prime \prime}\left(\lambda_{0}\right) u_{k}=0, \quad k \geq 0 .
\end{aligned}
$$

The dimension of $\mathcal{E}_{L}\left(\lambda_{0}\right)$ is the multiplicity of $\lambda_{0}$ (for details see [33]).

Assume that $L_{0}, L_{1}$ are self-adjoint, $L_{0}$ is positive non degenerate and that there exist $\kappa \geq 0$ and $\delta \geq 0$ such that $L_{1} L_{0}^{\delta-1 / 2}$ is a bounded operator on $\mathcal{H}$ and $\left\|L_{1} L_{0}^{\delta-1 / 2}\right\| \leq \kappa$. Assume that $L_{0}^{-1}$ is in the Schatten class $C^{p}, p \geq 1$.

Theorem 2.2. If $0<\delta \leq 1 / 2$ then the spectra of $L$ is the domain :

$$
\Omega_{\delta}=D_{R} \cup\left\{\lambda \in \mathbb{C},|\Re \lambda| \leq \kappa|\lambda|^{1-2 \delta}\right\}
$$

and $\oplus_{\lambda \in \operatorname{Sp}[L]} \mathcal{E}_{L}(\lambda)$ is dense in $\mathcal{H}$. If $\delta=0$ and if $\left|\frac{\pi}{2}-\arccos \kappa\right| \leq \frac{\pi}{2 p}$ then $\oplus_{\lambda \in \operatorname{Sp}[L]} \mathcal{E}_{L}(\lambda)$ is also dense in $\mathcal{H}$.

For $\delta>0$ we get that the eigenvalues are localized in a vertical parabolic domain in the imaginary direction. For $\delta=0$ end $\kappa$ small the eigenvalues are localized in a small sector around the imaginary axis. Notice that for $\kappa$ of order 1 the above theorem does not give any information on the location of $\mathrm{Sp}[L]$; we only know that it is a discrete and infinite subset of $\mathbb{C}$.

Moreover if $L_{1}$ has a sign we have :

Proposition 2.1. If $L_{1} \geq 0$ then $\operatorname{Sp}[L] \subseteq\{\lambda \in \mathbb{C}, \Re \lambda \leq 0\}$. If $L_{1} \leq 0$ then $\operatorname{Sp}[L] \subseteq\{\lambda \in \mathbb{C}, \Re \lambda \geq 0\}$.

The above result applies for example to :

$$
L(\lambda)=-\frac{d^{2}}{d x^{2}}+x^{6}+\alpha x^{2} \lambda+\lambda^{2}
$$

For this example we have $\delta=\frac{5}{6}$ hence the spectra is localized inside the parabolic region $\left\{\lambda \in \mathbb{C},|\Im \lambda| \geq C|\Re \lambda|^{5 / 2}\right\}$.

For Schrödinger pencils $L_{V, a}$ we can say more. 


\subsection{More results for Schrödinger pencils}

Let us recall our definition of Schrödinger pencils $L_{V, a}(\lambda)=-\triangle+V-2 a \lambda+\lambda^{2}$.

We assume that the pair of functions $(V, a)$ satisfies the following technical conditions that we refer as $[\operatorname{cond}(V, a)]$ (we do not try here to discuss the optimality of these conditions) :

$V, a$ are smooth $C^{\infty}$ functions on $\mathbb{R}^{d}$ and there exists $k>0$ such that

$$
\begin{aligned}
& \left|\partial_{x}^{\alpha} V(x)\right| \leq C_{\alpha}\langle x\rangle^{2 k-|\alpha|}, \quad\left|\partial_{x}^{\alpha} a(x)\right| \leq C_{\alpha}\langle x\rangle^{k-|\alpha|}, \\
& |a(x)| \leq \sqrt{V(x)}, \quad V(x) \geq 0, \quad V(x) \geq c\langle x\rangle^{2 k},
\end{aligned}
$$

for $|x| \geq 1$ with $\langle x\rangle:=\sqrt{1+x^{2}}$ and $C_{\alpha}>0, c>0$. Note that on a bounded domain, only the condition $|a(x)| \leq \sqrt{V(x)}$ has to be considered.

Under these conditions we know that $L_{0}=-\triangle+V$ is an unbounded self-adjoint operator in $L^{2}\left(\mathbb{R}^{d}\right)$ and for every $\lambda \in \mathbb{C} L_{V, a}(\lambda)$ is a closed and Fredholm operator with the following weighted Sobolev space domain:

$$
\mathcal{H}_{V}=\left\{u \in L^{2}\left(\mathbb{R}^{d}\right), \triangle u \in L^{2}\left(\mathbb{R}^{d}\right), V u \in L^{2}\left(\mathbb{R}^{d}\right)\right\} .
$$

Moreover the set $\operatorname{Sp}\left[L_{V, a}\right]$ of eigenvalues of $L_{V, a}$ is a discrete set (empty or not), each eigenvalue having a finite multiplicity and the only possible accumulation point in the complex plane is $\infty$.

Notice that if $\lambda$ is an eigenvalue then its complex conjugate $\bar{\lambda}$ is also an eigenvalue. We have the following result :

Proposition 2.2. Assume that $(V, a)$ satisfies $[\operatorname{cond}(V, a)]$ and that $a \leq 0, a\left(x^{0}\right)<0$ for some $x^{0} \in \mathbb{R}^{d}$. Then $\operatorname{Sp}\left[L_{V, a}\right]$ is in the open sector $\{\lambda \in \mathbb{C} ; \Im \lambda \neq 0, \Re \lambda \leq 0\}$.

Proof. Let $u \in L^{2}\left(\mathbb{R}^{d}\right), u \neq 0$ such that $L_{V, a}(\lambda) u=0$. Set $\lambda=r+i$. We know that $r \geq 0$. Assume that $r=0$. Reasoning by contradiction we first prove that $s=0$. If $s \neq 0$ we get that $\int_{\mathbb{R}^{d}} a(x)|u|^{2}(x) d x=$ hence $u$ vanishes in an non empty open set of $\mathbb{R}^{d}$ and applying the uniqueness Calderon theorem for second order elliptic equation we get $u=0$ on $\mathbb{R}^{d}$ and a contradiction. If $s=0$ we get :

$$
\left(-\triangle+V-2 r a+r^{2}\right) u=0
$$

and $\left.\int_{\mathbb{R}^{d}}\left(V(x)-2 r a(x)+r^{2}\right)\right)|u(x)|^{2} d x=0$. Using that $V \geq a^{2}$ we have

$$
\int_{\mathbb{R}^{d}}(r-a)^{2}|u(x)|^{2} d x=0 .
$$

So again we get that $u$ vanishes on a non empty open set and a contradiction like above. 
Let us remark that the general results given in Theorem 2.2 apply if there exists $\delta \geq 0$ such that $|a|(x) \leq C V(x)^{1 / 2-\delta}$ or $|a(x)| \leq \kappa V(x)^{1 / 2}$ with $\kappa$ small enough.

For 1D Schödinger pencils accurate results were obtained by M. Christ $[14,15]$ and by [13]. Let us recall here some of their results. They consider the pencils

$$
L_{k}(\lambda)=-\frac{d^{2}}{d x^{2}}+\left(x^{k}-\lambda\right)^{2}
$$

with $k \in \mathbb{N}$. Here we shall only consider $k$ even. The above assumptions are satisfied. We have :

Proposition 2.3 ([16]). For every $k \geq 2, k$ even, the set $\mathrm{Sp}\left[L_{k}\right]$ is included in the two sectors $\left\{\lambda \in \mathbb{C},|\arg (\lambda)| \geq \frac{k \pi}{2(k+1)}\right\}$.

The second result say that the eigenvalues of large modulus are close to the lines $\left\{\lambda \in \mathbb{C},|\arg (\lambda)|=\frac{k \pi}{2(k+1)}\right.$.

Theorem 2.3 ([13], Theorem 1). Let $\left\{\lambda_{n}\right\}_{n \in \mathbb{N}}$ be the set $\operatorname{Sp}\left[L_{k}\right]$ such that $\left|\lambda_{1}\right|<\cdots<\left|\lambda_{n}\right|<$ $\left|\lambda_{n+1}\right|<\cdots$. Then we have for $n \rightarrow+\infty$ :

$$
\lambda_{n}=\left(\frac{ \pm\left(n+\frac{1}{2}\right) \pi i-\log (2)}{\frac{2 k}{k+1}}\right)^{\frac{k}{k+1}}+O\left(\frac{1}{\sqrt{n}}\right) .
$$

This result was proved using ODE methods in the complex plane. Computing the argument for the complex number in the r.h.s of (2.2) we can see that $\left|\arg \left(\lambda_{n}\right)\right|$ is close to $\frac{k \pi}{2(k+1)}$ when $n \rightarrow+\infty$. We also have the following result :

Theorem 2.4 ([33], [1]). The linear space spanned by the generalized eigenfunctions associated with the eigenvalues $\left\{\lambda_{n}\right\}$ is dense in $L^{2}(\mathbb{R})$.

In [33] the proof was given for $L_{2}(\lambda)$ and for $L_{k}(\lambda), k>2$ and $k$ even in [1], [2].

In the following result we shall see that the spectral set $\operatorname{Sp}\left(L_{k}\right)$ is very unstable under perturbations. M. Christ [15] has considered the following model :

$$
L_{P}^{\#}(\lambda)=\left(P-\lambda+\frac{d}{d x}\right)\left(P-\lambda-\frac{d}{d x}\right) .
$$

We also have

$$
L_{P}^{\#}(\lambda)=-\frac{d^{2}}{d x^{2}}+(P-\lambda)^{2}+P^{\prime},
$$

where $P$ is a polynomial. Assume that the degree $k$ of $P$ is even, $P(x)=x^{k}+a_{k-1} x^{k-1}+$ $\cdots+a_{1} x+a_{0}$. We have :

Proposition 2.4. $\operatorname{Sp}\left[L_{P}^{\#}\right]=\varnothing$. In other words for every $\lambda \in \mathbb{C}$, the equation $L_{P}^{\#}(\lambda) u=0$ has only the trivial solution $u \equiv 0 \ddagger$ in the Schwartz space $\mathcal{S}(\mathbb{R})$.

$\ddagger_{\text {it }}$ is known that every solution in $L^{2}(\mathbb{R})$ of $L_{P}^{\#}(\lambda) u=0$ is in the Schwartz space $\mathcal{S}(\mathbb{R})$ (see [33]) 


\section{Spectra approximation with spectral methods and FDM}

The aim of this section is to present different numerical methods to analyze the localization in the complex plane of generalized spectrum of operator pencils like (2.1). More precisely we compare our numerical results with the theoretical results recalled in Section 2. Proposition 2.6 gives two sectors where one can find eigenvalues; Theorem 2.7 gives more accurate results on the localization for the eigenvalues with large modulus (when the modulus increases the eigenvalues are near the imaginary axis). The theoretical results of Section 2 are given in an infinite domain. But for numerical computations it is often needed to truncate the domain (for example for the finite difference method) for working in a finite domain. Then the question is to optimize the size of the domain to reduce the error term. This may be a delicate problem. Indeed the size of the domain can change dramatically the spectrum of the considered operator. For example taking $k=1$ in (2.1) we can see that the spectrum is empty in the infinite domain $\mathbb{R}$. But considering any finite interval and homogeneous Dirichlet conditions one can prove that the spectrum of (2.1) is discrete and infinite.

For the kind of pencils of differential operators considered in this paper the eigenfunctions are fast decreasing at infinity. But an eigenfunction $\varphi_{\lambda}$ associated with the complex eigenvalue $\lambda$ is small only if $|x| \geq f(\Im \lambda)$ for some increasing function $f$ such that $\lim _{r \rightarrow \infty} f(r)=\infty$. So we have to consider larger and larger domain to capture eigenvalues with large imaginary part.

In a first step we are staying with an infinite domain and we develop a spectral method using Hermite functions. This method is very accurate for our problem because the eigenfunctions are in the Schwartz space $\mathcal{S}(\mathbb{R})$.

In a second step we consider the finite domain approximation (with homogeneous Dirichlet conditions) and apply a finite difference method or a spectral Legendre-Galerkin method. As already said we have to optimize the size of the domain. This is solved by using properties of the zeros of Hermite functions.

Notice that the spectrum of the considered operator pencils is the spectrum of nonself-adjoint operators which are far from self-adjoint or normal operators. So the spectrum is very instable and it seems useful and important to compare different numerical methods. We also compare for our models the numerical computations of the spectrum with computations of the pseudo-spectrum.

Hereafter we present numerical methods for the computation of the spectra of linear operators with quadratic dependence of the spectral parameter $\lambda$ (quadratic pencil), see $(1.2)$ :

$$
L(\lambda)=L_{0}+\lambda L_{1}+\lambda^{2} \mathbb{I}
$$

where $L_{0}$ and $L_{1}$ are operators on some Hilbert space $\mathcal{H}$ and II is the identity operator. 


\subsection{A first quadratic pencil}

In this section we consider the following operator :

$$
L_{a}(\lambda)=-\frac{d^{2}}{d x^{2}}+x^{4}-2 a \lambda x^{2}+\lambda^{2},
$$

where $a$ is a real parameter. We want to solve the following nonlinear eigenvalue problem :

$$
L_{a}(\lambda) u=0, u \in L^{2}(\mathbb{R}), u \neq 0 .
$$

For $a=1$ we recover the problem (1.4). Notice that if $u$ satisfies (3.2) then we know that $u \in \mathcal{S}(\mathbb{R})$ (see [33]).

The problem (3.2) can be reformulated as an eigenvalue problem after linearization of $L_{a}(\lambda)$ (see Section 1). Indeed, if we set $v=\lambda u$ we can rewrite (3.2) as :

$$
\mathcal{A}_{a}\left(\begin{array}{l}
u \\
v
\end{array}\right)=\lambda\left(\begin{array}{l}
u \\
v
\end{array}\right)
$$

where

$$
\mathcal{A}_{a}=\left(\begin{array}{cc}
0 & \mathbb{I} \\
-L_{0} & -L_{1_{a}}
\end{array}\right)
$$

with the operators $L_{0}=-\frac{d^{2}}{d x^{2}}+x^{4}$ and $L_{1_{a}}=-2 a x^{2}$.

\subsubsection{Eigenvalue computations with Hermite spectral method (unbounded domain)}

We look for an approximation $u_{N}$ of $u$ such that $L_{a}(\lambda) u_{N}=0$, with :

$$
u_{N}=\sum_{k=0}^{N} \tilde{u}_{k} \varphi_{k}
$$

where $\varphi_{k}$ are Hermite functions (spectral Galerkin approximation, see Appendix A). The operator $L_{a}(\lambda)$ is defined on the domain $D(\mathcal{A})=\left\{u \in H^{2}(\mathbb{R}), x^{4} u \in L^{2}(\mathbb{R})\right\}$. Following (A.3) we deduce that the error $\left\|u-u_{N}\right\|$ decreases like $(2 N+1)^{-2}$ when $N$ increases. So it is not a priori necessary to retain a lot of terms in the development (3.4).

In order to compute $u_{N}$, we use a method of weighted residuals (MWR, see, e.g., ([10, 20]):

$$
\left(L_{a}(\lambda) u_{N}, \varphi_{l}\right)=0, \quad l=0, \ldots, N
$$

where $(.,$.$) is the scalar product in L^{2}(\mathbb{R})$. Setting $v_{N}=\lambda u_{N}$, using the orthogonality properties of the Hermite function in $L^{2}(\mathbb{R})$ and the relations (A.2), we obtain after linearization of $L(\lambda)$ the following eigenvalue problem :

$$
\mathcal{A}_{a, N}\left(\begin{array}{c}
U_{N} \\
V_{N}
\end{array}\right)=\lambda_{N}\left(\begin{array}{c}
U_{N} \\
V_{N}
\end{array}\right)
$$


which is an approximation of the eigenvalue problem (3.3). $U_{N}$ (resp. $V_{N}$ ) is the vector containing the coefficients $\tilde{u}_{k}$ (resp. $\tilde{v}_{k}$ ) of $u$ (resp. $v$ ), $k=0, \ldots, N$. The matrix $\mathcal{A}_{a, N}$ is the square matrix of order $(2 N+2)$ :

$$
\mathcal{A}_{a, N}=\left(\begin{array}{cc}
0 & \mathbb{I}_{N} \\
-L_{0_{N}} & -L_{1_{a, N}}
\end{array}\right)
$$

where $L_{0_{N}} u_{N}=\left(L_{0} u_{N}, \varphi_{l}\right)$ and $L_{a_{a, N}} v_{N}=\left(L_{1_{a}} v_{N}, \varphi_{l}\right), l=0, \ldots, N$, with

$$
L_{0} u_{N}=-\frac{d^{2} u_{N}}{d x^{2}}+x^{4} u_{N}, \quad L_{1_{a}} v_{N}=-2 a x^{2} v_{N} .
$$

Note that $L_{0_{N}}$ is a pentadiagonal symmetric matrix such that

$$
\begin{aligned}
& L_{0_{N}}(j, j)=\left(\frac{2 j+1}{2}\right)+\frac{1}{4} c_{j}, \quad L_{0_{N}}(j, j-4)=\frac{1}{4} a_{j-4} \\
& L_{0_{N}}(j, j-2)=-\frac{1}{2} \sqrt{j(j-1)}+\frac{1}{4} b_{j-2},
\end{aligned}
$$

for $j=0, \ldots, N$, where

$$
a_{j}=\sqrt{j(j-1)(j-2)(j-3)}, \quad b_{j}=(4 j-2) \sqrt{j(j-1)}, \quad c_{j}=\left(6 j^{2}+2 j+3\right) .
$$

Moreover, $L_{a, N}$ is a tridiagonal symmetric matrix such that

$$
L_{1_{a, N}}(j, j)=-(2 j+1), \quad L_{1_{a, N}}(j, j-2)=-\sqrt{j(j-1)} .
$$

For the numerical computation of the spectrum of $\mathcal{A}_{a, N}$ we use the function DGEEV of the LAPack library which is based on the Schur factorization.

For $a=1$, in order to analyze the spectrum of the continuous operator (1.4), we consider a simplified operator, deduced from the operator (3.1) for $a=1$, where $x$ is replaced with a real constant $b$. We obtain the following problem :

$$
\lambda^{2} u-\frac{d^{2} u}{d x^{2}}-2 b^{2} \lambda u+b^{4} u=0 .
$$

We look for a solution $u(x)$ of the problem (3.6) of the form $u=\tilde{u}_{k} \varphi_{k}, k=0, \ldots, N$. Substituting in (3.6) and using the relations (A.2), we obtain :

$$
\begin{aligned}
\lambda_{N}^{2} \varphi_{k}- & \frac{1}{2} \sqrt{k(k-1)} \varphi_{k-2}+\left(\frac{2 k+1}{2}\right) \varphi_{k}-\frac{1}{2} \sqrt{(k+1)(k+2)} \varphi_{k+2} \\
- & 2 b^{2} \lambda_{N} \varphi_{k}+b^{4} \varphi_{k}=0 .
\end{aligned}
$$

Using the scalar product in $L^{2}(\mathbb{R})$ of (3.7) with $\varphi_{k}$ gives

$$
\lambda_{N}^{2}+\frac{2 k+1}{2}-2 b^{2} \lambda_{N}+b^{4}=0 .
$$




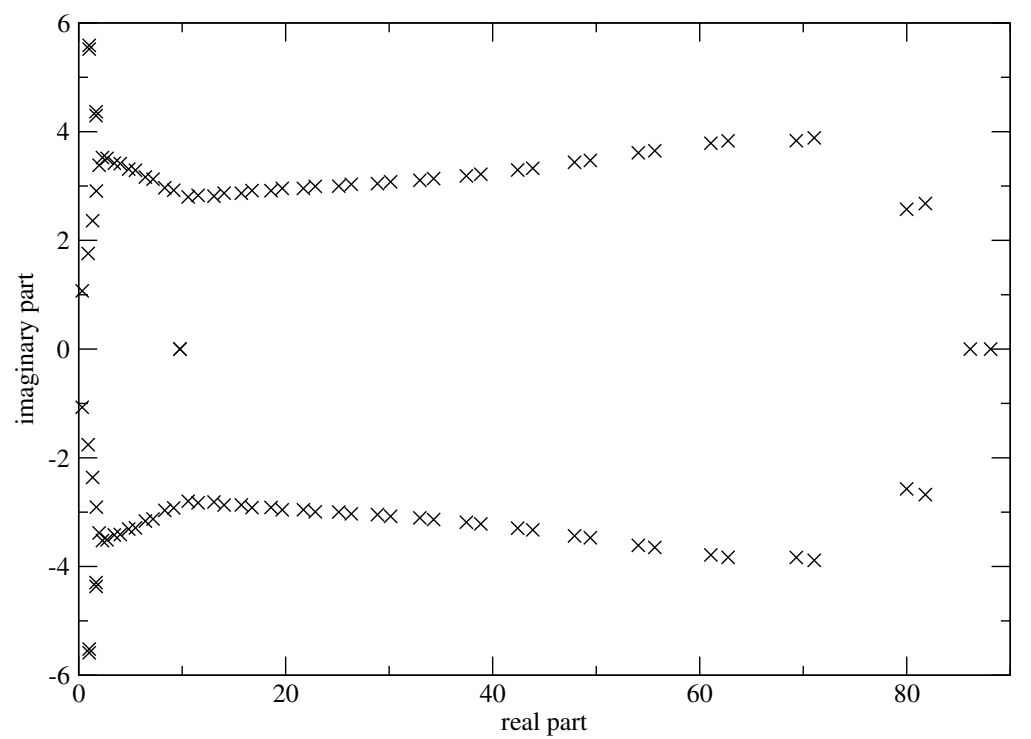

Figure 1: Spectrum of the matrix $\mathcal{A}_{a, N}$ (3.5) (Hermite spectral method) for $N=50$ and $a=1$.

We deduce from (3.8) that $\lambda_{N}=b^{2} \pm i \sqrt{k+1 / 2}$ that

$$
\lambda_{N, r}=b^{2}, \quad \lambda_{N, i}= \pm \sqrt{k+1 / 2} .
$$

The real part $\lambda_{N, r}$ of $\lambda_{N}$ is wavenumber independent and the imaginary part $\lambda_{N, i}$ is wavenumber dependent. From (3.9) we deduce that the spectrum is contained in the part of the complex plane defined by $\lambda_{N, r}=b^{2}$ and $-\sqrt{N} \leq \lambda_{N, i} \leq \sqrt{N}$ for $k=0, \ldots, N$.

Now, in Figure 1 we present the spectrum of the matrix (3.5) for $N=50$ and $a=1$. Firstly we can note that, as for the rotated harmonic oscillator (see [40]), a bifurcation appears in the spectrum when the modulus of the eigenvalues is increased (see also Figure 5). Then the theoretical results give that the eigenvalues of the continuous operator (3.1), for $a=1$, are included in the two sectors $\left\{\lambda \in \mathbb{C},|\arg (\lambda)| \geq \frac{\pi}{3}\right\}$ (see Section 2). We can see in Figure 1 that the computed eigenvalues are not all included in these two sectors. This reflects numerical instabilities leading to spurious eigenvalues (spectral pollution, see [18]).

We can note in Figure 1 that we have $-\sqrt{N} \leq \lambda_{N, i} \leq \sqrt{N}$, in agreement with the previous analyze when $x=b$ is constant (see (3.9)). Moreover, in the previous analyze we have $\lambda_{N, r}=b^{2}$. However if we retain $N$ modes in the Hermite development (3.4), the Hermite function of highest degree is $\varphi_{N}$ and the zeros $h_{n}$ of $\varphi_{N}$ verify (see [4]) :

$$
h_{n} \leq \sqrt{2 N-2}, \quad n=1, \ldots N .
$$

So the size of the containment area is

$$
2 L=2 \sqrt{2 N-2}
$$


and we can retain $b=L$ as value for $b$ in the estimation (3.9). Here, for $N=50$ following (3.10) we deduce that the size of the containment domain is $2 L$ with $L \simeq 10$. In Figure 1 , we can see that effectively we have $0 \leq \lambda_{N, r} \leq L^{2}$.

\subsubsection{Eigenvalue computations with finite difference method (bounded domain)}

The operator $L_{a}(\lambda)$ (see (3.1)) is defined on the domain $D(\mathcal{A})=\left\{u \in H^{2}(\mathbb{R}), x^{4} u \in L^{2}(\mathbb{R})\right\}$. So $u$ is decreasing as $O\left(1 / x^{4}\right)$ when $|x|$ is increasing (see (A.6)) and we want to consider the nonlinear eigenvalue problem (3.2) in a bounded domain with homogeneous Dirichlet boundary conditions : find $\lambda \in \mathbb{C}$ such that

$$
\left\{\begin{array}{l}
L_{a}(\lambda) u=0, \quad x \in \Omega, \\
u( \pm L)=0,
\end{array}\right.
$$

where $\Omega=(-L,+L)$ with $L$ sufficiently large. More precisely following (3.10) we retain $L=\sqrt{2 N-2}$.

As before, the problem (3.11) can be reformulated as an eigenvalue problem

$$
\mathcal{A}_{a}\left(\begin{array}{c}
u \\
v
\end{array}\right)=\lambda\left(\begin{array}{l}
u \\
v
\end{array}\right)
$$

where

$$
\mathcal{A}_{a}=\left(\begin{array}{cc}
0 & \mathbb{I} \\
-L_{0} & -L_{1_{a}}
\end{array}\right)
$$

with $v=\lambda u$ and the operators $L_{0}=-\frac{d^{2}}{d x^{2}}+x^{4}, L_{1_{a}}=-2 a x^{2}$.

To obtain an approximation of the continuous problem (3.12) in a finite dimensional space, we consider on the domain $\Omega$ a mesh-grid with a mesh $\Delta x=2 L / N$ on $\Omega$ and we note $x_{j}=-L+j \Delta x, j=0, \ldots N$ the points of the grid. We have retained homogeneous Dirichlet boundary conditions for $x= \pm L$, so $u\left(x_{0}\right)=u\left(x_{N}\right)=0$. We look for an approximation $u_{N}, v_{N}$ of $u$ and $v=\lambda u$ such that:

$$
\mathcal{A}_{a, N}\left(\begin{array}{c}
U_{N} \\
V_{N}
\end{array}\right)=\lambda_{N}\left(\begin{array}{c}
U_{N} \\
V_{N}
\end{array}\right)
$$

with $U_{N}$ and $V_{N}$ two vectors containing respectively the approximations $u_{N}\left(x_{j}\right), v_{N}\left(x_{j}\right)$ of $u\left(x_{j}\right), v\left(x_{j}\right)$ and $\mathcal{A}_{a, N}$ is the square matrix of order $2 N-2$ :

$$
\mathcal{A}_{a, N}=\left(\begin{array}{cc}
0 & \mathbb{I}_{N} \\
-L_{0_{N}} & -L_{1_{a, N}}
\end{array}\right)
$$

where

$$
L_{0_{N}} u_{N}\left(x_{j}\right)=-\frac{u_{N}\left(x_{j+1}\right)-2 u_{N}\left(x_{j}\right)+u_{N}\left(x_{j-1}\right)}{\Delta x^{2}}+x_{j}^{4} u_{N}\left(x_{j}\right)
$$


is the discretization of the operator $L_{0}$ with a second order centered finite difference scheme and

$$
L_{1_{a, N}} v_{N}\left(x_{j}\right)=-2 a x_{j}^{2} v_{N}\left(x_{j}\right) .
$$

$L_{0_{N}}$ is a tridiagonal symmetric matrix such that

$$
L_{0_{N}}(j, j)=\frac{2}{\Delta x^{2}}+x_{j}^{4}, \quad L_{0_{N}}(j, j-1)=-\frac{1}{\Delta x^{2}}
$$

and $L_{a, N}$ is a diagonal matrix such that $L_{1_{a, N}}(j, j)=-2 a x_{j}^{2}$.

For the numerical computation of the spectrum of the matrix $\mathcal{A}_{a, N}$ we use the function DGEEV of the LAPack library.

Now, we are interested to analyze the dependence of the spectrum of the operator (3.1) in function of the real parameter $a$. For this, we consider an approximation of the infinite dimensional domain as a bounded domain with periodic boundary conditions. We look for eigenfunction $u_{k}(x)=\hat{u}_{k} \exp \left(i k^{\prime} x\right)$, with $k^{\prime}=k \pi / L$, of the continuous operator (3.1). Computing $L_{a}(\lambda) u_{k}(x)$ we obtain the following equation :

$$
\lambda^{2}-2 a \lambda x^{2}+x^{4}+k^{\prime 2}=0 .
$$

The discriminant $\Delta=4\left(a^{2}-1\right) x^{4}-4 k^{\prime 2}$ is negative for $0 \leq a \leq 1$ and the solutions are :

$$
\lambda^{ \pm}=\frac{2 a x^{2} \pm i \sqrt{-\Delta}}{2}
$$

When $a$ is increased from 0 to 1 the ratio of the imaginary part over the real part of $\lambda$,

$$
\frac{\left|\lambda_{i}\right|}{\left|\lambda_{r}\right|}=\frac{\sqrt{k^{\prime 2}-\left(a^{2}-1\right) x^{4}}}{a x^{2}}
$$

is decreased and it is infinite for $a=0$. We can observed this on the numerical simulations corresponding to $a=0, a=0.5, a=0.9$ and $a=1.0$ obtained with the finite difference scheme for $N=50$ and $L=10$ (see Figure 2).

We are now interested with the operator $L_{a}(\lambda)$ for $a=1$. We look for the spectrum of the discretized operator, using finite difference method, where $x$ is replaced with a real constant $b$ as in (3.6). Indeed, when we discretize with a finite difference scheme, we consider that $x$ is constant over one spatial step $\Delta x=2 L / N$. So, in a first approximation, we consider an operator deduced from $L_{a}(\lambda)$ in which $x$ is chosen constant equal to $b$ over all the domain $\Omega$. We have :

$$
\lambda_{N}^{2} u\left(x_{j}\right)-\frac{u\left(x_{j+1}\right)-2 u\left(x_{j}\right)+u\left(x_{j-1}\right)}{\Delta x^{2}}-2 b^{2} \lambda_{N} u\left(x_{j}\right)+b^{4} u\left(x_{j}\right)=0 .
$$

If we consider periodic boundary conditions, we look for a solution of (3.14) of the form $u(x)=\hat{u}_{k} \exp \left(i k^{\prime} x\right)$, with $k^{\prime}=k \pi / L$. Substituting in (3.14) and supposing that $\hat{u}_{k} \neq 0$ we obtain

$$
\lambda_{N}^{2} \Delta x^{2}-2 b^{2} \lambda_{N} \Delta x^{2}+b^{4} \Delta x^{2}-2 \cos \left(k^{\prime} \Delta x\right)+2=0 .
$$




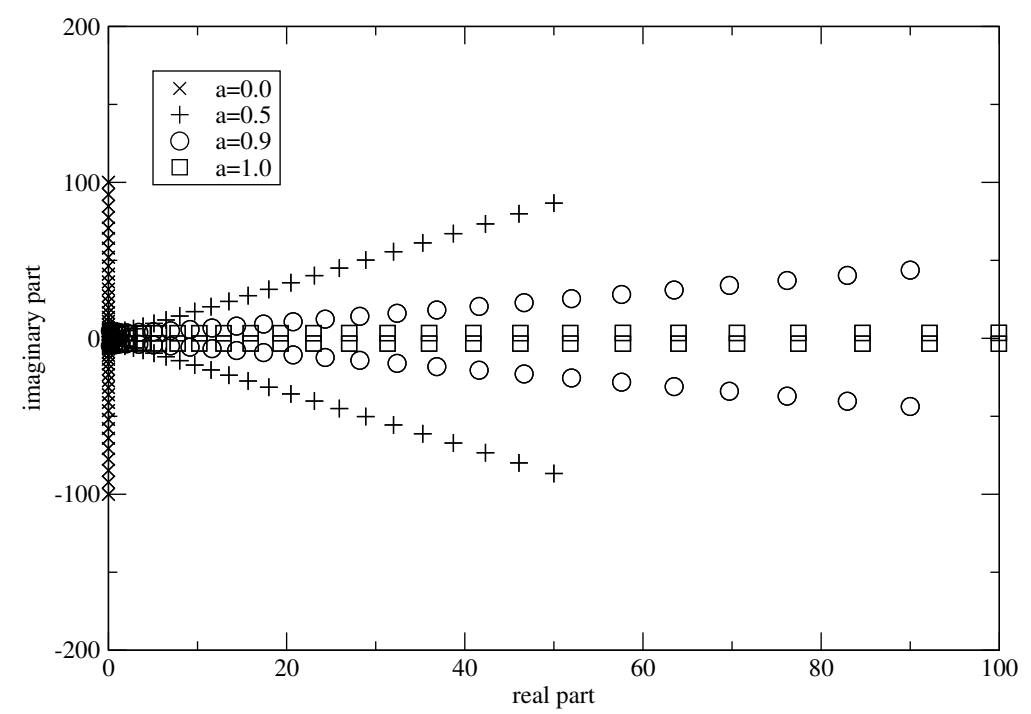

Figure 2: Spectrum obtained with the finite difference scheme for $a=0, a=0.5, a=0.9, a=1.0, N=50$ and $L=10$.

Finally we have $\lambda_{N}=\lambda_{N, r}+i \lambda_{N, i}$ with $\lambda_{N, r}=b^{2}$ is wavenumber independent and

$$
\lambda_{N, i}= \pm \frac{\sqrt{2-2 \cos \left(k^{\prime} \delta x\right)}}{\Delta x}
$$

is wavenumber dependent. So the spectrum of the discretized operator is located in the part of the complex plane such that

$$
\lambda_{N, r}=b^{2}, \quad-\left|k_{\max }^{\prime}\right| \leq \lambda_{N, i} \leq\left|k_{\max }^{\prime}\right|
$$

since $\cos \left(k^{\prime} \Delta x\right) \simeq 1-k^{\prime 2} \Delta x^{2} / 2$ for $\Delta x$ sufficiently small. Here since $\Omega=(-L,+L)$ and $N$ is the number of grid points retained, the highest wavenumber $k_{\max }^{\prime}$ we can take into account with this mesh-grid is

$$
k_{\max }^{\prime}=\frac{N}{2 L}=\Delta x^{-1} .
$$

Since $L \simeq \sqrt{2 N-2}$ (see (3.10)) we have $k_{\max }^{\prime}=O(\sqrt{N})$, so $-\sqrt{N} \leq \lambda_{N, i} \leq \sqrt{N}$ which is in agreement with the Hermite spectral method for unbounded domain (see (3.9)).

In Figure 3 we present the spectrum of the matrix (3.13) for $N=50, L=10$ and $a=1$. Comparison with Figure 1 shows that the results obtained for Hermite spectral method (unbounded domain) and for finite difference method (bounded domain) are quite similar. We have chosen $L=10$ for the size of the bounded domain, in agreement with (3.10). As it has been said previously, theoretical results give that the eigenvalues of the continuous operator (3.1), for $a=1$, are included in the two sectors $\left\{\lambda \in \mathbb{C},|\arg (\lambda)| \geq \frac{\pi}{3}\right\}$ (see Section 2). But we can see in Figure 3 (as in Figure 1) that computed eigenvalues are not all included in these two sectors, which can be imputed to numerical instabilities leading to spurious eigenvalues (spectral pollution, see [18]). 


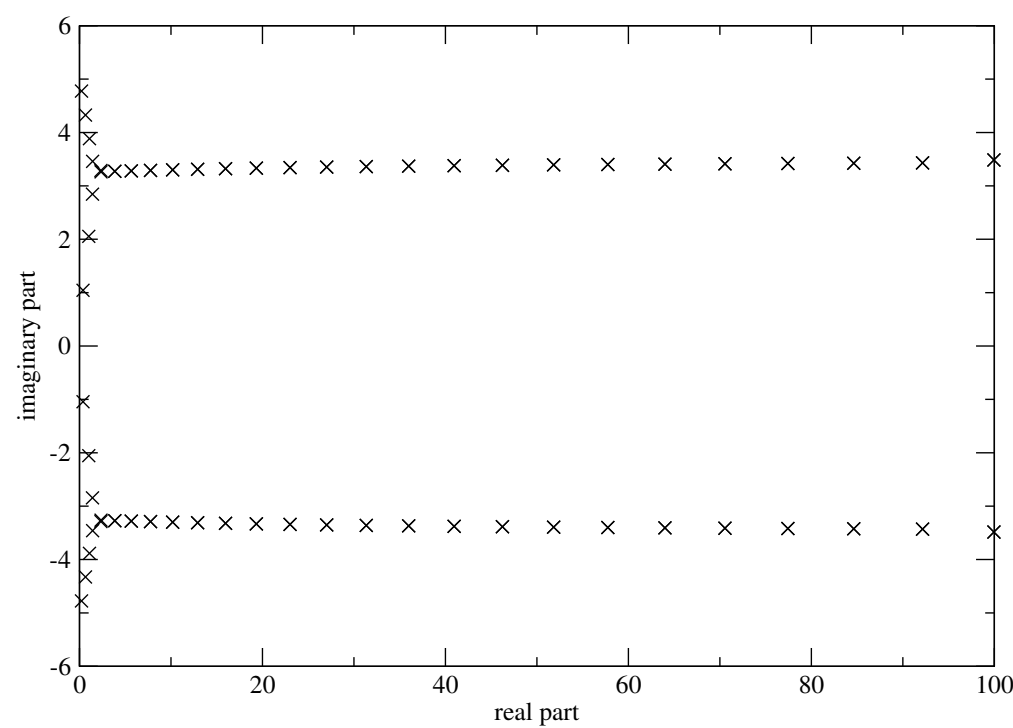

Figure 3: Spectrum of the matrix $\mathcal{A}_{a, N}$ (3.13) (finite difference scheme) for $N=50, L=10$ and $a=1$.

Remark 3.1. With the previous estimates obtain on the localization of the eigenvalues $\left(\lambda_{N, r}=b^{2}\right.$ and $\left.-\sqrt{N} \leq \lambda_{N, i} \leq \sqrt{N}\right)$, we can deduce that we have $|\lambda| \leq L^{2}$. Indeed, after finite difference discretization of the continuous operator (3.1) we can consider that $b$ is constant on each mesh of the grid with $b \simeq x_{i} \in(-L,+L)$. Now let us consider localization theorems for quadratic eigenvalue problems in finite dimension (see [6]). After discretization of the quadratic pencil (3.1) we obtain the matrix $T_{N}(\lambda)=D_{N}(\lambda)+E_{N}$, where $D_{N}(\lambda)$ is the diagonal matrix such that $D_{N}(j, j)=-2 a \lambda x_{j}^{2}+\lambda^{2}$ and $E_{N}$ is a tridiagonal symmetric matrix such that $E_{N}(j, j)=x_{j}^{4}-\frac{2}{\Delta x^{2}}$ and $E_{N}(j, j-1)=\frac{1}{\Delta x^{2}}$. Applying Theorem 3.1 in [6] we obtain the estimate

$$
\left|-2 a \lambda x_{j}^{2}+\lambda^{2}\right| \leq N\left(4 \Delta x^{2}+L^{4}\right)
$$

in agreement with $|\lambda| \leq L^{2}$.

\section{Numerical instability}

In order to study the numerical instability of the finite difference scheme in function of the mesh-grid $x_{j}, j=0, \ldots N$, we consider a small perturbation on each point of the grid, $x_{j}+\varepsilon, j=0, \ldots N$, where $\varepsilon$ is a small parameter. The matrix $\mathcal{A}_{a, N}$ (see (3.13)) is replaced with the matrix :

$$
\mathcal{A}_{a, N, \varepsilon}=\mathcal{A}_{a, N}+\varepsilon \mathcal{E}_{N}
$$

where $\mathcal{E}_{N}$ is a perturbation matrix. If we compare the eigenvalues $\lambda_{N}$ of the matrix $\mathcal{A}_{a, N}$ with the eigenvalues $\lambda_{N, \varepsilon}$ of $\mathcal{A}_{a, N, \varepsilon}$ we have :

$$
\mathcal{A}_{a, N, \varepsilon} U_{N, \varepsilon}=\left(\mathcal{A}_{a, N}+\varepsilon \mathcal{E}_{N}\right) U_{N, \varepsilon}=\lambda_{N, \varepsilon} U_{N, \varepsilon}
$$




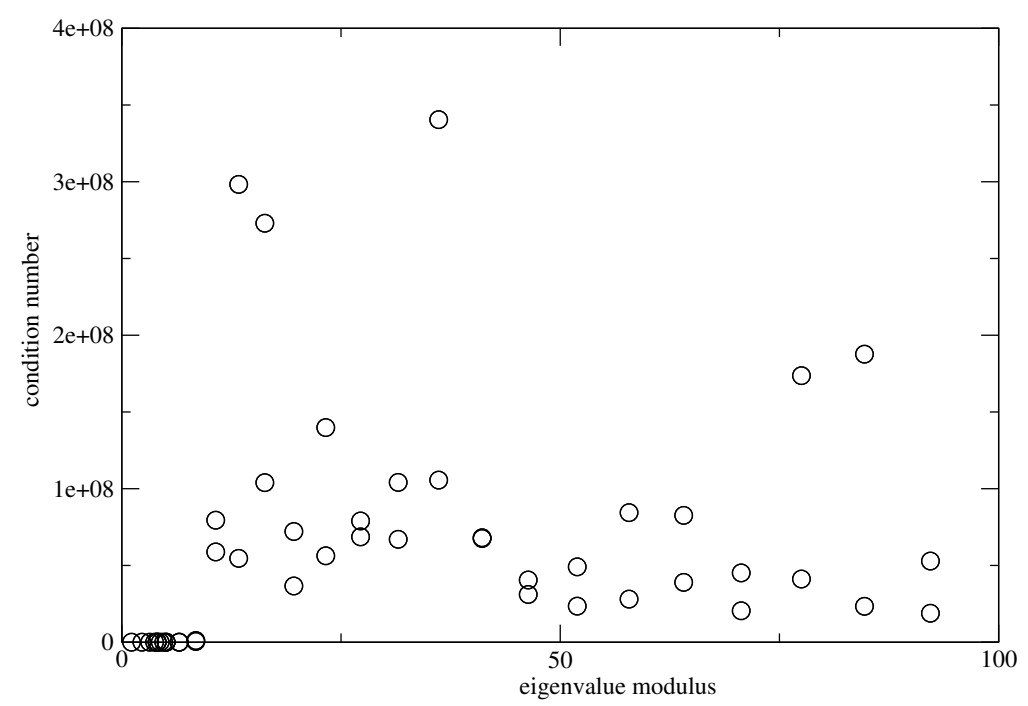

Figure 4: Condition number of the eigenvalues $\lambda_{N}$ in function of the modulus $\left|\lambda_{N}\right|$ for $N=50, L=10$ and $a=1$.

where $U_{N, \varepsilon}$ is a right eigenvector of $A_{a, N, \varepsilon}$. So we deduce (see [35]) :

$$
\mathcal{A}_{a, N} \frac{d U_{N, \varepsilon}}{d \varepsilon}(\varepsilon)+\mathcal{E}_{N} U_{N, \varepsilon}(\varepsilon)+\varepsilon \mathcal{E}_{N} \frac{d U_{N, \varepsilon}}{d \varepsilon}(\varepsilon)=\frac{d \lambda_{N, \varepsilon}}{d \varepsilon}(\varepsilon) U_{N, \varepsilon}(\varepsilon)+\lambda_{N, \varepsilon}(\varepsilon) \frac{d U_{N, \varepsilon}}{d \varepsilon}(\varepsilon) .
$$

For $\varepsilon=0$ we obtain :

$$
\mathcal{A}_{a, N} \frac{d U_{N, \varepsilon}}{d \varepsilon}(0)+\mathcal{E}_{N} U_{N, \varepsilon}(0)=\frac{d \lambda_{N, \varepsilon}}{d \varepsilon}(0) U_{N, \varepsilon}(0)+\lambda_{N, \varepsilon}(0) \frac{d U_{N, \varepsilon}}{d \varepsilon}(0)
$$

If we multiply on the left the previous equality with $V_{N}$ a left eigenvector of $\mathcal{A}_{a, N}$ we obtain :

$$
\frac{d \lambda_{N, \varepsilon}}{d \varepsilon}(0)=\frac{V_{N}^{\star} \mathcal{E}_{N} U_{N}}{V_{N}^{\star} U_{N}}
$$

where $V_{N}^{\star}=\bar{V}_{N}{ }^{t}$ and $U_{N}$ is a right eigenvector of $\mathcal{A}_{a, N}$. The equality (3.15) measures the sensitivity of the eigenvalue $\lambda_{N}$ of the matrix $\mathcal{A}_{a, N}$ in function of a perturbation $\varepsilon$ on the mesh-grid (condition number of the eigenvalue $\lambda_{N}$ ).

Here the matrix $\mathcal{E}_{N}$ is the matrix of order $2 N-2$ :

$$
\mathcal{E}_{N}=\varepsilon\left(\begin{array}{cc}
0 & 0 \\
\mathcal{E}_{0, N} & \mathcal{E}_{1, N}
\end{array}\right)
$$

where $\mathcal{E}_{0, N}$ (resp. $\left.\mathcal{E}_{1, N}\right)$ is the diagonal matrix with the elements $-4 x_{j}^{3}$ (resp. $4 a x_{j}$ ) on the diagonal, $j=1, \ldots, N-1$ (we have neglected in $\mathcal{E}_{N}$ the terms in $\varepsilon^{n}$, with $n>1$ ).

Figure 4 presents the condition number of the eigenvalues $\lambda_{N}$ in function of the modulus $\left|\lambda_{N}\right|$ for $N=50, L=10$ and $a=1$. We can see that eigenvalues are ill conditioned, 


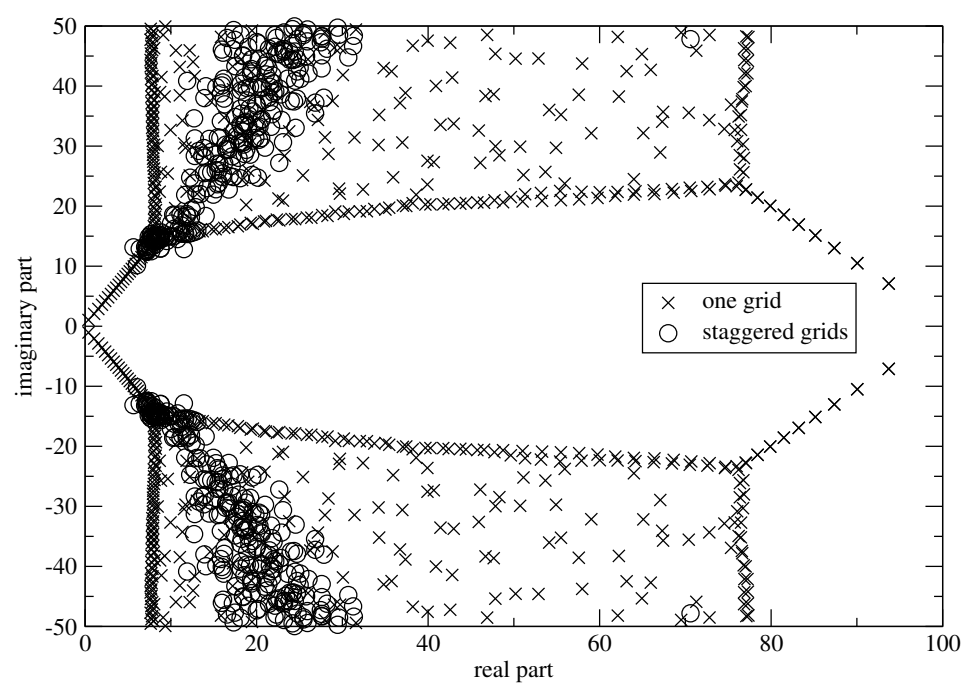

Figure 5: Average on the eigenvalues computed with the finite difference scheme using 11 staggered grids for $a=1, N=1000$ and $L=10$.

excepted for the eigenvalues with small modulus. This can explain the convergence problem when $N$ is increased. If we compare with the rotated harmonic oscillator the condition numbers of the eigenvalues are much greater for the operator (3.1) (around $10^{8}$ ) than for the rotated harmonic oscillator (around 10). A small perturbation on the grid points induces large perturbations on the eigenvalue computations.

However the eigenvalues are independent of $x$. So, in order to decrease this dependence of the eigenvalues in function of a perturbation on the points of the mesh grid, we have considered several grids for the finite difference discretization, with a shift on the mesh points, but with the same step $\Delta x$ for the mesh grid : $y_{j}=x_{j}+\varepsilon$. Then we compute an average on the eigenvalues obtained with these staggered grids. The results obtained are presented in Figure 5, which corresponds to $a=1, N=1000$ and $L=10$. The number of staggered grids retained is 11 . We can see that spurious eigenvalues have disappeared. The computed eigenvalues after averaging are now essentially contained in the area $\left\{\lambda \in \mathbb{C},|\arg (\lambda)| \geq \frac{\pi}{3}\right\}$ in agreement with theoretical results (see Section 2). We can note in Figure 5 that on the imaginary axis we have limited the imaginary part of $\lambda_{N}$ to $\left|\lambda_{N, i}\right| \leq N / 2 L=50$. Indeed, as its has been said previously, $\lambda_{N, i}$ is function of the wavenumber and the highest wavenumber we can take into account on the grids is $N / 2 L$.

For the use of staggered meshes to avoid spectral pollution, we may mentioned the following reference [29].

\section{Pseudospectra}

Now we consider the pseudospectra since it is known that the numerical computation of the pseudospectra is more stable than for the spectra (see Appendix B). To obtain the 


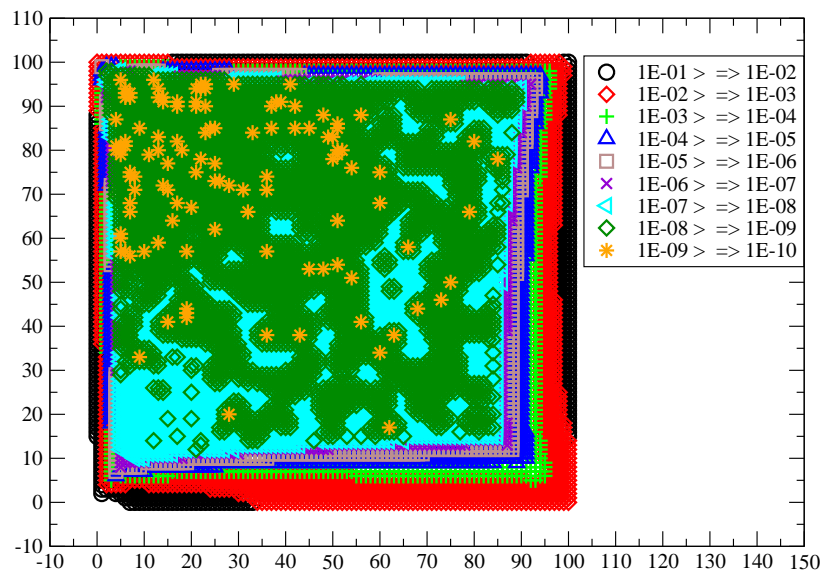

Figure 6: Computation of the pseudospectra (3.16) of the matrix $\mathcal{A}_{a, N}$ (3.13) (finite difference scheme), for $N=1000, L=10$ and $a=1$. The different colors correspond to different size of the small parameter $\varepsilon$. The smallest parameter $\varepsilon$ corresponds to orange snowflakes.

pseudospectra, following Definition B.2 we look for $z \in \mathbb{C}$ such that $\left\|\left(\mathcal{A}_{N}-z \mathbb{I}_{N}\right)^{-1}\right\|=$ $s_{\min }^{-1}\left(\mathcal{A}_{N}-z \mathbb{I}_{N}\right)$ is large, i.e. :

$$
s_{\min }\left(\mathcal{A}_{N}-z \mathbb{I}_{N}\right) \leq \varepsilon,
$$

where $\varepsilon$ is a small parameter; $\frac{1}{\varepsilon}$ is a lower bound for the norm of the resolvent $\|\left(\mathcal{A}_{N}-\right.$ $\left.z \mathbb{I}_{N}\right)^{-1} \|$ at the point $z \in \mathbb{C}$. For the computation of the pseudospectra, we have retained complex values $z$ in (3.16) lying on the mesh-grid in the part of the complex plane corresponding to $[0,100] \times[0,100]$. The step retained is $d x=d y=1$ in the real and imaginary directions. On Figure 6 we can see the computation for the matrix $\mathcal{A}_{a, N}-z \mathbb{I}_{N}$ with $\mathcal{A}_{a, N}$ corresponding to the matrix (3.13), for $N=1000, L=10$ and $a=1$. The different colors in Figure 6 correspond to different size of the small parameter $\varepsilon$. We can note that, in agreement with the theoretical results (see Section 2), the two sectors $\left\{\lambda \in \mathbb{C},|\arg (\lambda)| \geq \frac{\pi}{3}\right\}$ of the spectrum of the continuous operator (3.1) are essentially contained in the area of the pseudospectra corresponding to the small parameter $\varepsilon$, i.e. in the area where the distance of $z$ to the eigenvalues of the matrix (3.13) is the smallest.

When the mesh-grid retained in the complex plane is fine, the pseudospectra computation is expensive since it requires to compute the minimal singular value $s_{\min }\left(\mathcal{A}_{N}-z \mathbb{I}_{N}\right)$ at each point $z$ of the mesh. So we use parallel computation in order to accelerate the computation. The numerical solution is done thanks to the linear algebra library LAPack which contains specialized algorithms for singular values problems, especially the one called ZGESVD for complex matrices in double precision (ZGESVD is based on bidiagonal QR iteration). As the matrix (3.13) is quite huge, and computing time a bit long, a parallelization by MPI (Message Passing Interface) is implemented with the client/server model. One process (the server) distributes values of the complex parameter $z$ (see (3.16)) 
to the other processes (the clients) which sample the domain. The server renews their data as the work progresses. Each client builds the matrix to be study and sends to the server, at the end of the computation, the smallest value. This system has the advantage of being dynamically balanced. As there is no communication (in MPI sense) between the clients, the efficiency of the parallelization is complete. As an example, the simulation corresponding to the parameters $N=5000, L=1000, a=1$ and to an area of the complex plane $[0,150] \times[0,150]$ with a mesh step $d x=1$ and $d y=1$ in the real and imaginary directions has needed 40 cores (Intel Xeon E5-2670 at 2.5GHz) during quite 40 days.

\subsubsection{Eigenvalue computations with Legendre spectral Galerkin method}

In order to obtain a higher accurate numerical scheme in bounded domain, we propose a spectral numerical scheme using Legendre Galerkin basis.

We consider the problem (3.11). This problem is reformulated as an eigenvalue problem (3.12). But instead of using a finite difference scheme to obtain an approximation $u_{N}$, $v_{N}$ of $u$ and $v=\lambda u$, we use a spectral method with Legendre Galerkin basis $\Phi_{l}$. Such basis is obtained as a linear combination of Legendre polynomials :

$$
\Phi_{l}(x)=c_{l}\left(L_{l}(x)-L_{l+2}(x)\right)
$$

with $L_{l}$ the Legendre polynomial of degree $l$ and $c_{l}=\frac{1}{\sqrt{4 l+6}}$ (see [27]). Such a basis verify homogeneous Dirichlet boundary conditions $\Phi_{l}( \pm 1)=0$. In particular, with the scalar product in $L_{2}(\Omega)$ we have :

$$
\left(\Phi_{k}, \Phi_{j}\right)= \begin{cases}c_{k} c_{j}\left(\frac{2}{2 j+1}+\frac{2}{2 j+5}\right), & k=j \\ -c_{k} c_{j} \frac{2}{2 k+1}, & k=j+2 \\ 0, & \text { otherwise }\end{cases}
$$

and

$$
\left(\Phi_{k}^{\prime}, \Phi_{j}^{\prime}\right)= \begin{cases}1, & k=j \\ 0, & k \neq j .\end{cases}
$$

Moreover, we need the expressions of $x^{2} \Phi_{l}$ and $x^{4} \Phi_{l}$ as linear combination of the Legendre polynomials. We have :

$$
\begin{array}{r}
x^{2} L_{l}(x)=\zeta_{l}\left(\frac{l+1}{2 l+3}\left((l+2) L_{l+2}(x)+(l+1) L_{l}(x)\right)\right. \\
\left.+\frac{l}{2 l-1}\left(l L_{l}(x)+(l-1) L_{l-2}(x)\right)\right)
\end{array}
$$

where $\zeta_{l}=\frac{1}{2 l+1}$ and

$$
x^{4} L_{l}(x)=\alpha_{l} L_{l-4}(x)+\beta_{l} L_{l-2}(x)+\gamma_{l} L_{l}(x)+\delta_{l} L_{l+2}(x)+\eta_{l} L_{l+4}(x),
$$


with

$$
\begin{aligned}
& \alpha_{l}=\zeta_{l}\left(\frac{l(l-1)(l-2)(l-3)}{(2 l-1)(2 l-3)(2 l-5)}\right) \\
& \beta_{l}=\zeta_{l}\left(\left(\frac{(l+1)^{2}}{(2 l+3)(2 l+1)}+\frac{l^{2}}{(2 l-1)(2 l+1)}\right)\left(\frac{l(l-1)}{2 l-1}\right)+\frac{l(l-1)^{3}}{(2 l-1)^{2}(2 l-3)}+\frac{l(l-1)(l-2)^{2}}{(2 l-1)(2 l-3)(2 l-5)}\right), \\
& \gamma_{l}=\zeta_{l}\left(\left(\frac{(l+1)^{2}(l+2)^{2}}{(2 l+3)^{2}(2 l+5)}+\left(\frac{(l+1)^{2}}{(2 l+1)(2 l+3)}+\frac{l^{2}}{(2 l-1)(2 l+1)}\right) *\left(\frac{(l+1)^{2}}{(2 l+3)}+\frac{l^{2}}{(2 l-1)}\right)+\frac{l^{2}(l-1)^{2}}{(2 l-1)^{2}(2 l-3)}\right),\right. \\
& \delta_{l}=\zeta_{l}\left(\left(\frac{(l+1)^{2}}{(2 l+3)(2 l+1)}+\frac{l^{2}}{(2 l-1)(2 l+1)}\right)\left(\frac{(l+1)(l+2)}{2 l+3}\right)+\frac{(l+1)(l+2)^{3}}{(2 l+3)^{2}(2 l+5)}+\frac{(l+1)(l+2)(l+3)^{2}}{(2 l+3)(2 l+5)(2 l+7)}\right), \\
& \eta_{l}=\zeta_{l}\left(\frac{(l+1)(l+2)(l+3)(l+4)}{(2 l+3)(2 l+5)(2 l+7)}\right) .
\end{aligned}
$$

In order to adapt the previous basis $\Phi_{l}$ to the Dirichlet boundary conditions $\Phi_{l}( \pm L)=0$, we multiply the previous polynomials by a scale factor. As for the Hermite spectral method (see Section 3.2.1), we use a method of weighted residuals (MWR, see, e.g., [10, 20]) and relations (3.17)-(3.20) to obtain the following generalized eigenvalue problem :

$$
\mathcal{A}_{a, N}\left(\begin{array}{c}
U_{N} \\
V_{N}
\end{array}\right)=\lambda_{N} \mathcal{B}_{N}\left(\begin{array}{c}
U_{N} \\
V_{N}
\end{array}\right)
$$

where $U_{N}$ and $V_{N}$ are the vectors containing respectively the coefficients $\tilde{u}_{l}$ and $\tilde{v}_{l}, l=$ $0, \ldots, N$, of $u_{N}=\sum_{l=0}^{N} \tilde{u}_{l} \Phi_{l}$ and $v_{N}=\sum_{l=0}^{N} \tilde{v}_{l} \Phi_{l} . \mathcal{A}_{a, N}$ is the square matrix of order $(2 N+2)$ :

$$
\mathcal{A}_{a, N}=\left(\begin{array}{cc}
0 & \mathbb{I}_{N} \\
-L_{0_{N}}^{\prime} & -L_{1_{a, N}}^{\prime}
\end{array}\right)
$$

and $\mathcal{B}_{N}$ is the square matrix of order $(2 N+2)$ :

$$
\mathcal{B}_{N}=\left(\begin{array}{cc}
B_{0_{N}} & 0 \\
0 & B_{0_{N}}
\end{array}\right)
$$

Here $L_{0_{N}}^{\prime} u_{N}=\left(L_{0} u_{N}, \Phi_{l^{\prime}}\right)$ and $L_{1_{a, N}}^{\prime} v_{N}=\left(L_{1_{a}} v_{N}, \Phi_{l^{\prime}}\right), l^{\prime}=0, \ldots, N$, with

$$
L_{0} u_{N}=-\frac{d^{2} u_{N}}{d x^{2}}+x^{4} u_{N}, \quad L_{1_{a}} u_{N}=-2 a x^{2} u_{N}
$$

As for $B_{0_{N}} u_{N}=\left(u_{N}, \Phi_{l^{\prime}}\right)$. Note that $L_{0_{N}}^{\prime}$ is a symmetric matrix with seven diagonal and $L_{1_{a, N}}^{\prime}$ is a pentadiagonal symmetric matrix. As for $B_{0_{N}}=\left(\Phi_{l}, \Phi_{l^{\prime}}\right)$ for $l$ and $l^{\prime}=0, \ldots N$ (see (3.17)).

To obtain the eigenvalues of the generalized eigenvalue problem (3.21) we use the function DGGEV of the LAPack library which is based on the generalized Schur factorization. 


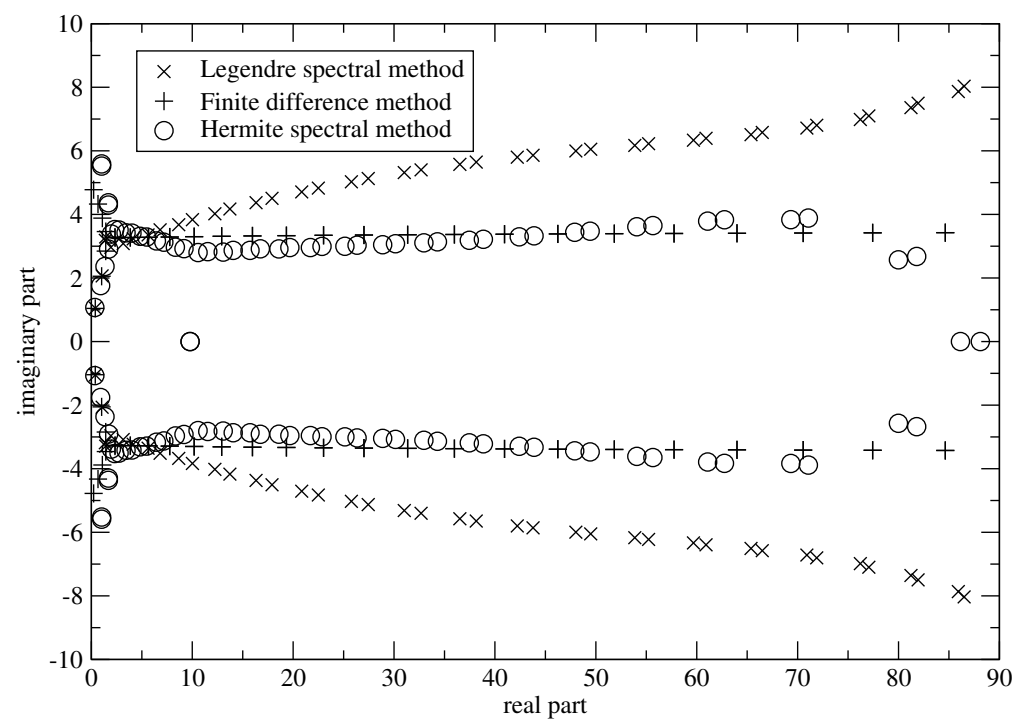

Figure 7: Computation of the eigenvalues for $N=50, L=10$ and $a=1$ with the Legendre spectral method. Comparison with the spectral Hermite method and the finite difference method is done.

In Figure 7 we present the solutions $\lambda_{N}$ of (3.21), computed with $N=50, L=10$ and $a=1$. Comparison with the spectral Hermite method (Figure 1) and the finite difference method (Figure 3 ) is done. We can see that the numerical results are quite similar.

\subsection{Another quadratic operator}

In this section we consider the following operator :

$$
\mathcal{L} u(x)=-\frac{d^{2} u}{d x^{2}}(x)+\left(x^{k}-\lambda\right)^{2} u(x) .
$$

For $k=2$ we retrieve the operator (1.4) studied in the previous section.

We discretize the problem $\mathcal{L} u=0$ using some techniques similar to finite difference methods, with a spatial step equal to one. For simplicity reasons we need to add either periodic boundary conditions or homogeneous Dirichlet boundary conditions. Also we replace $\Delta u(n)$ by $\delta \delta^{*}$ where:

$$
\delta u(n)=u(n+1)-u(n), \quad \delta^{*} u(n)=u(n)-u(n-1), \quad n \in \mathbb{N},
$$

i.e.

$$
\left(\delta \delta^{*}\right) u(n)=u(n-1)-2 u(n)+u(n+1)
$$

So we have :

$$
\mathcal{L} u(n) \approx-\left(\delta \delta^{*}\right) u(n)+\left(n^{k}-\lambda\right)^{2} u(n), \quad n \in \mathbb{N} .
$$




\subsubsection{Finite difference method with periodic boundary conditions}

In this section we are interested to study the problem (3.23) with periodic boundary conditions. So for some $N \in \mathbb{N}$, we study the following problem :

$$
\begin{array}{ll}
-\left(\delta \delta^{*}\right) u(n)+\left(n^{k}-\lambda\right)^{2} u(n)=0, & n=1, \cdots, N, \\
u(j)=u(j+N), & j=0,1 .
\end{array}
$$

This gives the following quadratic eigenvalue problem :

$$
A_{0}+\lambda A_{1}+\lambda^{2} \mathbb{I}=0
$$

where II is the $N \times N$ identity matrix and $A_{1}, A_{0}$ are given as follows :

$$
\begin{aligned}
A_{1} & =-2\left(\begin{array}{ccccccc}
1 & 0 & \cdots & & & \cdots & 0 \\
0 & 2^{k} & 0 & \cdots & & \cdots & 0 \\
\vdots & & \vdots & & \vdots & & \vdots \\
0 & \cdots & & \cdots & 0 & (N-1)^{k} & 0 \\
0 & \cdots & & & \cdots & 0 & N^{k}
\end{array}\right), \\
A_{0} & =A_{0, d}+A_{0,+1}+A_{0,-1},
\end{aligned}
$$

with

$$
\begin{aligned}
& A_{0, d}=\left(\begin{array}{cccccccccc}
2+1 & 0 & 0 & \cdots & & & & \cdots & 0 & 0 \\
0 & 2+2^{2 k} & 0 & 0 & \cdots & & & & \cdots & 0 \\
& & & \vdots & & & \vdots & & & \\
0 & \cdots & \cdots & 0 & 0 & 2+j^{2 k} & 0 & 0 & \cdots & 0 \\
\vdots & & & \vdots & & & \vdots & & & \vdots \\
0 & \cdots & & & & \cdots & 0 & 0 & 2+(N-1)^{2 k} & 0 \\
0 & 0 & \cdots & & & & \cdots & 0 & 0 & 2+N^{2 k}
\end{array}\right), \\
& A_{0,+1}=\left(\begin{array}{ccccccc}
0 & -1 & \cdots & & & \cdots & -1 \\
0 & 0 & -1 & \cdots & & \cdots & 0 \\
\vdots & & \vdots & & \vdots & & \vdots \\
0 & \cdots & & \cdots & 0 & 0 & -1 \\
0 & \cdots & & & \cdots & 0 & 0
\end{array}\right), A_{0,-1}=\left(\begin{array}{ccccccc}
0 & 0 & \cdots & & & \cdots & 0 \\
-1 & 0 & 0 & \cdots & & \cdots & 0 \\
0 & -1 & 0 & \cdots & & \cdots & 0 \\
\vdots & & \vdots & & \vdots & & \vdots \\
0 & \cdots & & \cdots & 0 & 0 & 0 \\
-1 & \cdots & & & \cdots & -1 & 0
\end{array}\right) .
\end{aligned}
$$

We start by computing the eigenvalues for different values of $N$ and for the operator $\mathcal{L}$. Then, we compute the eigenvalues for some perturbations of the operator $\mathcal{L}$, i.e. we study the discrete operator :

$$
\mathcal{L}_{c} u(n)=A_{0} u(n)+c \lambda A_{1} u(n)+\lambda^{2} \mathbb{I} u(n), \quad n=1, \cdots, N
$$



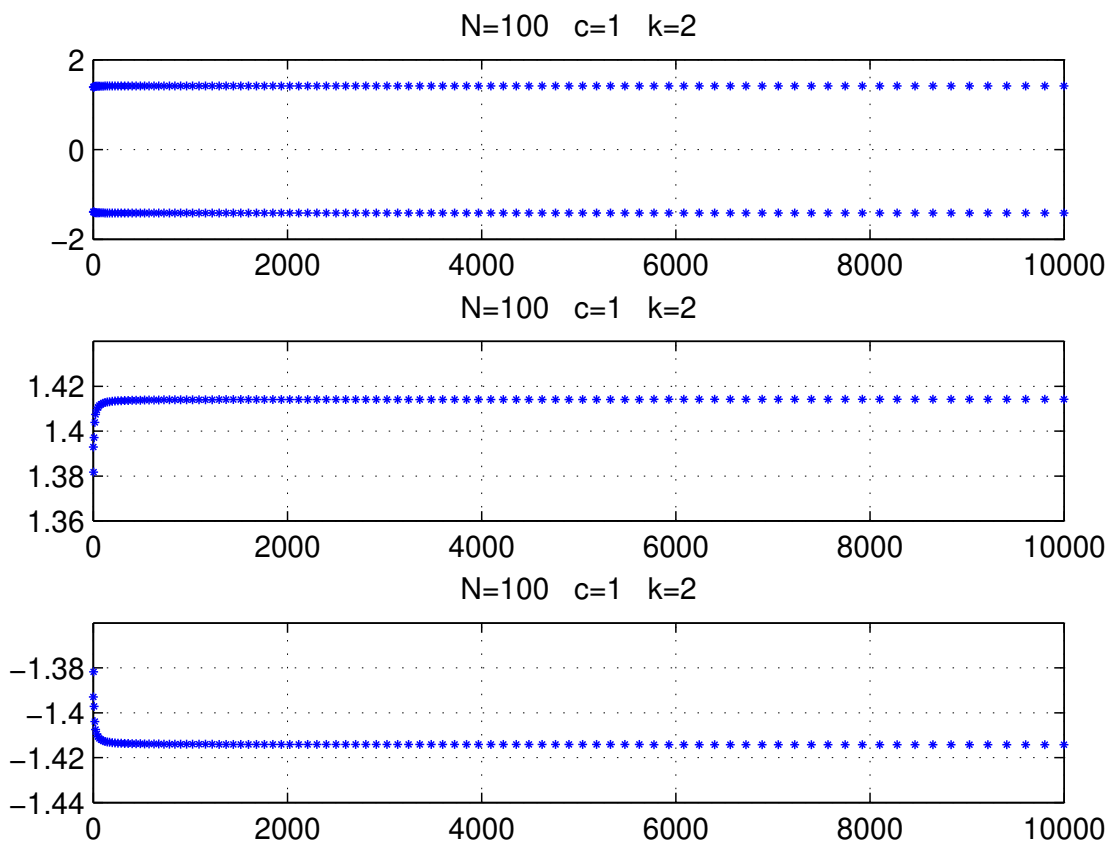

Figure 8: Eigenvalues of the matrix $\mathcal{A}_{c}$ for $N=100, c=1$ and $k=2$. This figure represents a zoom for the case $c=1$.

for $0 \leq c \leq 1$ with the same previous periodic boundary conditions. For this we consider the linearization system problem in place of the non-linear problem, so we study the spectrum of the linear system $\mathcal{A}_{c} U=\lambda U$ with :

$$
\mathcal{A}_{c}=\left(\begin{array}{cc}
0 & \mathbb{I} \\
-A_{0} & -c A_{1}
\end{array}\right), \quad 0 \leq c \leq 1,
$$

where $U=\left(u_{1}, u_{2}, \cdots, u_{N-1}, u_{N}, v_{1}, v_{2}, \cdots, v_{N-1}, v_{N}\right)^{t}$, with $v_{i}=\lambda u_{i}, i=1, \cdots, N$. $A_{0}$ and $A_{1}$ are given in (3.25) and (3.24) respectively. For the computation of the eigenvalues, we use Matlab.

The results obtained for $N=100 k=2$ and $c=1$ are presented in Figure 8 . The associated domain is $[0, N]$. This figure represents a zoom for the case $c=1$. We note that the imaginary part of the eigenvalues $\lambda_{i}$ lies between 1.38 and 1.42 in the positive part and between -1.42 and -1.38 in the negative part. Starting from a real part $\lambda_{r}=576$ all the eigenvalues are aligned on a straight parallel to the $x$-axis with $\lambda_{i}=1.4141$ and $\lambda_{i}=-1.4141$. The results obtained for $N=1000 k=2$ and $c=1$ are similar.

In Figure 9 we present the numerical results obtained for $N=1000, k=4$ and $0 \leq c \leq$ 1. For the case $c=0$ we have pure imaginary eigenvalues (since in this case we have just a selfadjoint matrix). The positions of eigenvalues for the cases $c=0.2,0.4$ confirm 

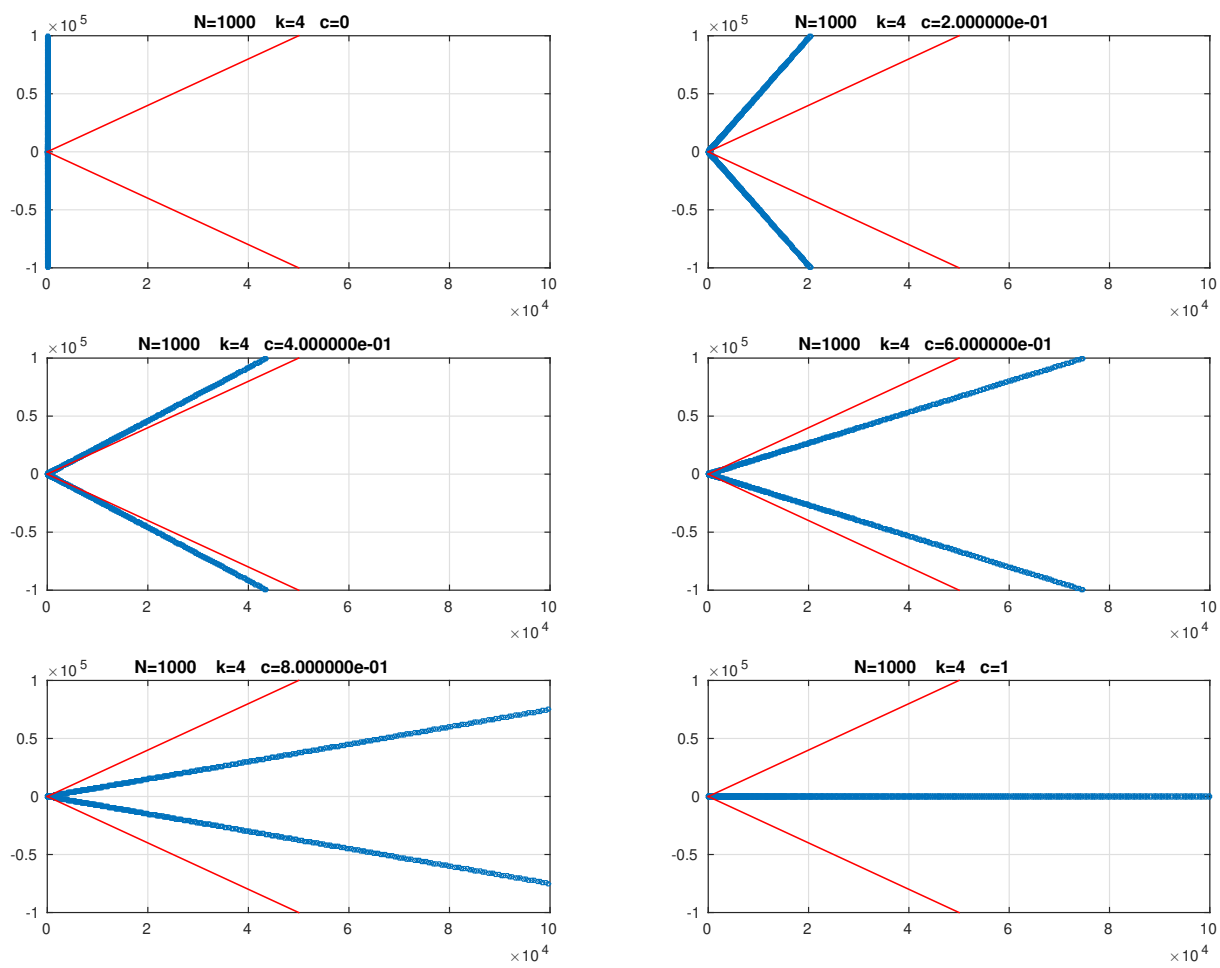

Figure 9: Eigenvalues of the matrix $\mathcal{A}_{c}$ for $N=1000, k=4$ and $c=0,0.2,0.4,0.6,0.8,1$. In the first three figures we can see the cases $c=0,0.2,0.4$. In the last three figures we can see the cases $c=0.6,0.8,1$.

the theoretical results. For the cases $c=0.6,0.8,1$, eigenvalues are localized in a sector delimited by an angle with the $x$-axis smaller than $2 \pi / 6$. This is not coherent with the theoretical results.

\subsubsection{Finite difference method with homogeneous Dirichlet boundary conditions}

Now we consider the problem (3.23) with homogeneous Dirichlet boundary conditions. So we study the following problem :

$$
\begin{aligned}
& -\left(\delta \delta^{*}\right) u(n)+\left(n^{k}-\lambda\right)^{2} u(n)=0, \quad n=1, \ldots, N, \\
& u(0)=u(N+1)=0 .
\end{aligned}
$$

We obtain the following nonlinear eigenvalue problem :

$$
A_{0}+\lambda A_{1}+\lambda^{2} \mathbb{I}=0,
$$

where $\mathbb{I}$ is the $N \times N$ identity matrix and $A_{1}, A_{0}$ are given as follows : 

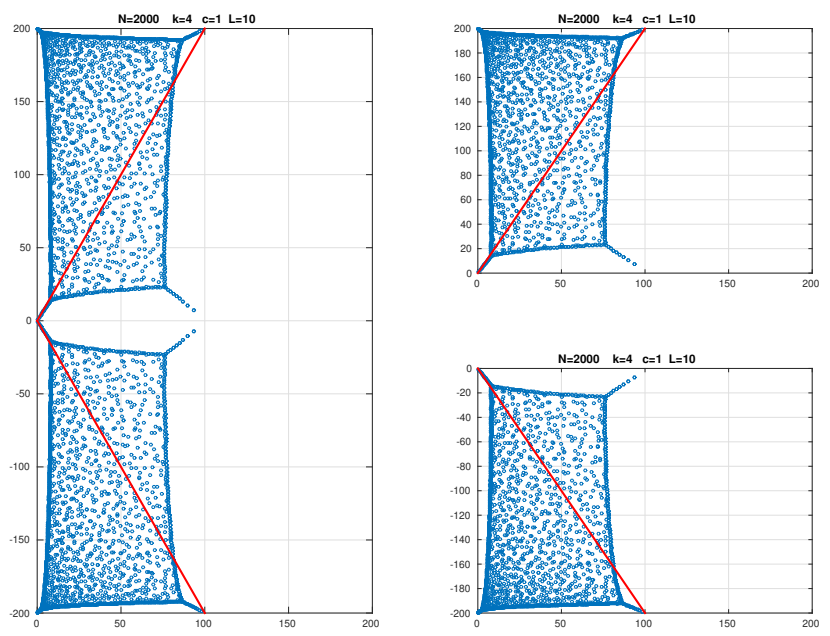

Figure 10: Eigenvalues of the matrix $\mathcal{A}_{c}$ for $N=2000, k=4, L=10, c=1$.

$$
\begin{aligned}
A_{1} & =-2\left(\begin{array}{ccccccc}
1 & 0 & \cdots & & & \cdots & 0 \\
0 & 2^{k} & 0 & \cdots & & \cdots & 0 \\
\vdots & & \vdots & & \vdots & & \vdots \\
0 & \cdots & & \cdots & 0 & (N-1)^{k} & 0 \\
0 & \cdots & & & \cdots & 0 & N^{k}
\end{array}\right), \\
A_{0} & =A_{0, d}+A_{0,+1}+A_{0,-1},
\end{aligned}
$$
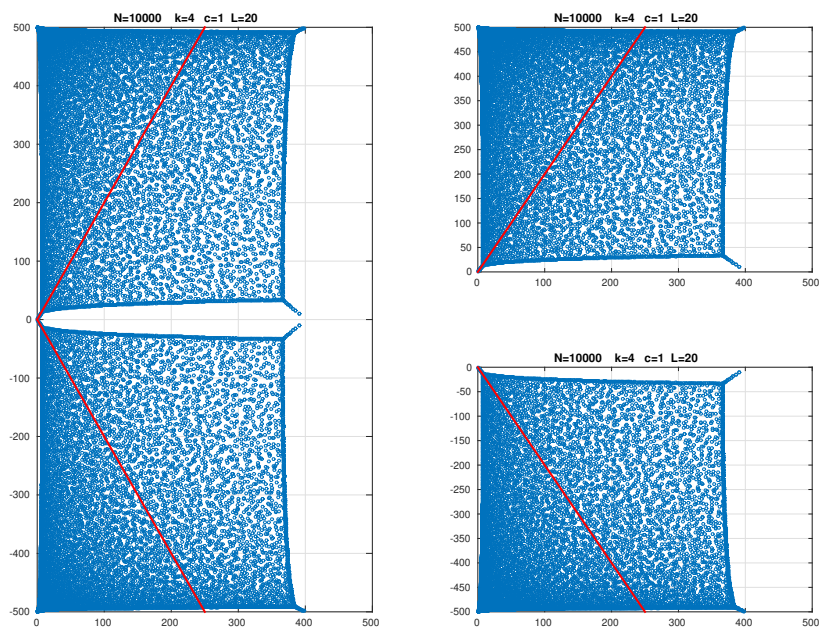

Figure 11: Eigenvalues of the matrix $\mathcal{A}_{c}$ for $N=10000, k=4, L=20, c=1$. 
where

$$
\begin{aligned}
& A_{0, d}=\left(\begin{array}{cccccccccc}
2+1 & 0 & 0 & \cdots & & & & \cdots & 0 & 0 \\
0 & 2+2^{2 k} & 0 & 0 & \cdots & & & & \cdots & 0 \\
& & & \vdots & & & \vdots & & & \\
0 & \cdots & \cdots & 0 & 0 & 2+j^{2 k} & 0 & 0 & \cdots & 0 \\
\vdots & & & \vdots & & & \vdots & & & \vdots \\
0 & \cdots & & & & \cdots & 0 & 0 & 2+(N-1)^{2 k} & 0 \\
0 & 0 & \cdots & & & & \cdots & 0 & 0 & 2+N^{2 k}
\end{array}\right), \\
& A_{0,+1}=\left(\begin{array}{ccccccc}
0 & -1 & \cdots & & & \cdots & 0 \\
0 & 0 & -1 & \cdots & & \cdots & 0 \\
\vdots & & \vdots & & \vdots & & \vdots \\
0 & \cdots & & \cdots & 0 & 0 & -1 \\
0 & \cdots & & & \cdots & 0 & 0
\end{array}\right), A_{0,-1}=\left(\begin{array}{ccccccc}
0 & 0 & \cdots & & & \cdots & 0 \\
-1 & 0 & 0 & \cdots & & \cdots & 0 \\
0 & -1 & 0 & \cdots & & \cdots & 0 \\
\vdots & & \vdots & & \vdots & & \vdots \\
0 & \cdots & & \cdots & 0 & 0 & 0 \\
0 & \cdots & & & \cdots & -1 & 0
\end{array}\right) .
\end{aligned}
$$

We start by computing the eigenvalues for different values of $N$ and for the operator $\mathcal{L}$. Then we compute the eigenvalues for some perturbations of the operator $\mathcal{L}$, i.e. we consider the discrete operator :

$$
\mathcal{L}_{c} u(n)=A_{0} u(n)+c \lambda A_{1} u(n)+\lambda^{2} \mathbb{I} u(n), \quad n=1, \cdots, N
$$

with $0 \leq c \leq 1$ and the same previous homogeneous Dirichlet boundary conditions. We do this considering the linearization system problem in place of the non-linear problem. So we study the spectrum of the linear system $\mathcal{A}_{c} U=\lambda U$ with :

$$
\mathcal{A}_{c}=\left(\begin{array}{cc}
0 & \mathbb{I} \\
-A_{0} & -c A_{1}
\end{array}\right), \quad 0 \leq c \leq 1,
$$

where $U=\left(u_{1}, u_{2}, \cdots, u_{N-1}, u_{N}, v_{1}, v_{2}, \cdots, v_{N-1}, v_{N}\right)^{t}$, with $v_{i}=\lambda u_{i}, i=1, \cdots, N$. $A_{0}$ and $A_{1}$ are given in (3.28) and (3.27) respectively. We compute the eigenvalues using Matlab.

For the numerical simulations we have considered a domain $[-L,+L]$ and a spatial step $\Delta x=2 L / N$. For the case $k=4$, the results obtained for $L=10, N=2000$ (resp. $L=20$, $N=10000$ ) and $c=1$ are presented in Figures 10 and 11 respectively. For the case $k=6$, the numerical results obtained for the example (3.26b) with $N=10000, c=1$ and $L=20$ (resp. $L=10$ ) are presented in Figure 12 and 13 respectively.

Remark 3.2. We can note that when the parameter $k$ is increased, the numerical results obtained are in better agreement with the theoretical results given in Section 2, i.e. the eigenvalues of the continuous operator (3.22) are included in the two sectors $\left\{\lambda \in \mathbb{C},|\arg (\lambda)| \geq \frac{k \pi}{2(k+1)}\right\}$. This can be explained by the fact that the eigenvalues are better conditioned when $k$ is increased. 

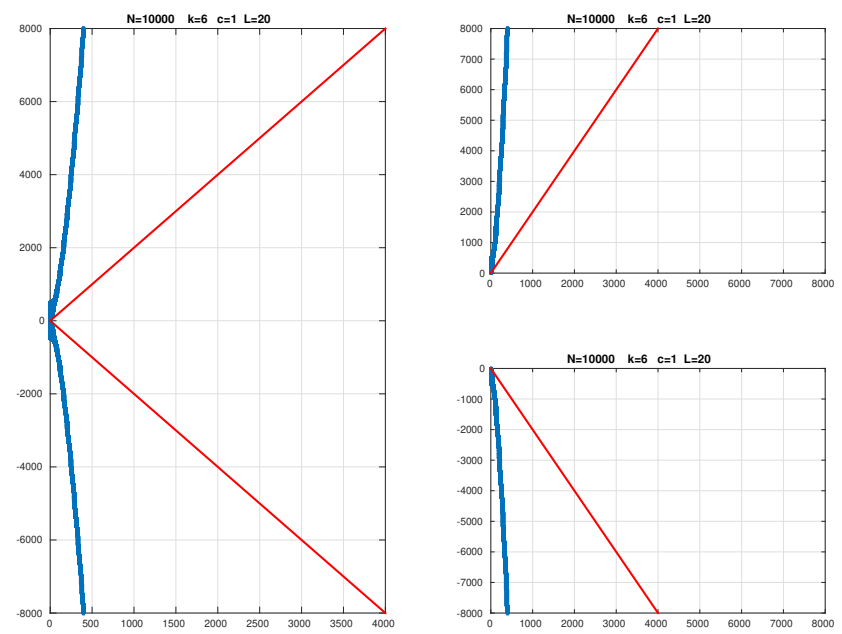

Figure 12: Eigenvalues of the matrix $\mathcal{A}_{c}$ for $N=10000, k=6, L=20, c=1$. The figure on the left corresponds to $0 \leq \Re \lambda \leq 10000$ and $-10000 \leq \Im \lambda \leq 10000$. The two figures on the right correspond, up to $0 \leq \Re \lambda \leq 10000$ and $0 \leq \Im \bar{\lambda} \leq 10000$, down to $0 \leq \Re \lambda \leq 10000$ and $-10000 \leq \Im \lambda \leq 0$.
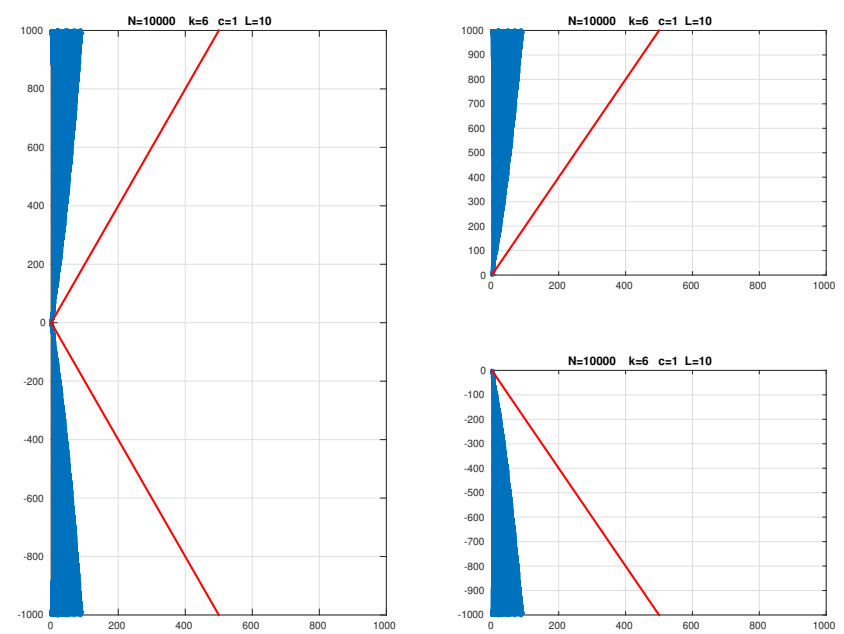

Figure 13: Eigenvalues of the matrix $\mathcal{A}_{c}$ for $N=10000, k=6, L=10, c=1$. The figure on the left corresponds to $0 \leq \Re \lambda \leq 1500$ and $-1500 \leq \Im \lambda \leq 1500$. The two figures on the right correspond, up to $0 \leq \Re \lambda \leq 1500$ and $0 \leq \Im \bar{\varsigma} \leq 1500$, down to $0 \leq \Re \bar{\lambda} \leq 1500$ and $-1500 \leq \Im \lambda \leq 0$.

\section{Conclusions and open problems}

In this work we have presented a review of some theoretical results obtained for quadratic family of operators :

$$
L(\lambda)=L_{0}+\lambda L_{1}+\lambda^{2}
$$


where $L_{0}$ and $L_{1}$ are operators in an Hilbert space.

Then we have presented numerical methods to compute the spectrum of such operators. We reduce it to a non self-adjoint linear eigenvalue problem. The numerical methods proposed are based on spectral methods and finite difference methods, in bounded and unbounded domains. For bounded domain we consider homogeneous Dirichlet boundary conditions and periodic boundary conditions. Comparison of the results obtained in unbounded and bounded domains are done. They are based on the size of the containment domain, deduces from the zeros of the Hermite functions.

The numerical results obtained are presented. In particular the numerical instabilities are highlighted. Comparisons of the numerical results obtained in Section 2, with the theoretical results presented in Section 2, are done. These comparisons show the difficulties for the numerical computation of the spectra of such operators. Elimination of the spectral pollution, using staggered grids, and the computation of pseudospectra allow to obtain numerical results in agreement with theoretical results.

A future step in this work is the extension to higher dimension (two and three dimensional case). Indeed, in the multidimensional case very few results are known on the location of the eigenvalues. Numerical simulations, using numerical approaches developed and validate in this article, and completed with parallel computing, should allow to locate eigenvalues. This work is in progress and will be presented elsewhere.

\section{Acknowledgments}

This work was initiated during the visit of Fatima Aboud, at the Laboratoire de Mathématiques Jean Leray, Université de Nantes (France), CNRS UMR 6629. This visit was supported by the research project DéfiMaths of the Fédération de Mathématiques des Pays de la Loire, CNRS FR 2962. F.A. was also supported by the CIMPA (International Center of Pure ans Applied Mathematics). Computations are done thanks to the computer of the CCIPL (Centre de Calcul Intensif des Pays de la Loire).

\section{Appendix A Hermite spectral approximation}

The basis $\left\{\varphi_{k}\right\}_{k \in \mathbb{N}}$ of Hermite functions is obtained as an orthonormal basis of $L^{2}(\mathbb{R})$ of the eigenfunctions of the harmonic oscillator :

$$
H_{o s c}=-\frac{d^{2}}{d x^{2}}+x^{2}
$$

We recall briefly its construction (see the basic books of quantum mechanics). Define the creation operator $a^{*}$ and the annihilation operator $a$ :

$$
a^{*}=x-\frac{d}{d x}, \quad a=x+\frac{d}{d x} .
$$


We satisfy :

$$
\left[a, a^{*}\right]=2 \mathbb{I}, \quad H_{o s c}=a^{*} a+\mathbb{I}=\frac{1}{2}\left(a a^{*}+a^{*} a\right),
$$

where $\left[a, a^{*}\right]=a a^{*}-a^{*} a$.

Starting by the normalized Gaussian :

$$
\varphi_{0}(x)=\pi^{-1 / 4} \mathrm{e}^{-x^{2} / 2}
$$

verified $a \phi_{0}=0$ and then $H_{o s c} \varphi_{0}=\varphi_{0}$ one define by induction for integer $k$ the sequence $\left\{\varphi_{k}\right\}_{k \in \mathbb{N}}$ :

$$
\begin{gathered}
\varphi_{k+1}=(2(k+1))^{-1 / 2} a^{*} \varphi_{k} \\
\Rightarrow \varphi_{k}=2^{-k / 2}(k !)^{-1 / 2}\left(a^{*}\right)^{k} \varphi_{0} .
\end{gathered}
$$

We verify the following relation by using an algebraic calculation :

$$
\begin{aligned}
& a \varphi_{k+1}=(2 k+1)^{1 / 2} \varphi_{k}, \quad a^{*} \varphi_{k}=(2 k+1)^{1 / 2} \varphi_{k+1}, \\
& H_{o s c} \varphi_{k}=(2 k+1) \varphi_{k}, \quad\left\langle\varphi_{k}, \varphi_{\ell}\right\rangle=\delta_{k, \ell},
\end{aligned}
$$

where $\langle$,$\rangle denoted the scalar product in the (complex) Hilbert space L^{2}(\mathbb{R})$. We then show that $\left\{\varphi_{k}\right\}_{k \in \mathbb{N}}$ is a Hilbertian basis of $L^{2}(\mathbb{R})$.

To do the projection of the differential operators in this basis we need to calculate the multiplication by $x$ and the derivation $\frac{d}{d x}$ of $\varphi_{k}$. We use the relations $x=\frac{a+a^{*}}{2}$ and $\frac{d}{d x}=\frac{a-a^{*}}{2}$. By the relations (A.1) we obtain :

$$
\begin{aligned}
& x \varphi_{k}=2^{-1 / 2} \sqrt{k} \varphi_{k-1}+\sqrt{k+1} \varphi_{k+1}, \\
& \frac{d}{d x} \varphi_{k}=2^{-1 / 2} \sqrt{k} \varphi_{k-1}-\sqrt{k+1} \varphi_{k+1} \text {, } \\
& x^{2} \varphi_{k}=\frac{1}{2}\left(\sqrt{k(k-1)} \varphi_{k-2}+(2 k+1) \varphi_{k}+\sqrt{(k+1)(k+2)} \varphi_{k+2}\right), \\
& x^{4} \varphi_{k}=\frac{1}{4}\left(\sqrt{k(k-1)(k-2)(k-3)} \varphi_{k-4}+(4 k-2) \sqrt{k(k-1)} \varphi_{k-2}\right. \\
& +\left(6 k^{2}+2 k+3\right) \varphi_{k}+(4 k+6) \sqrt{(k+1)(k+2)} \varphi_{k+2} \\
& \left.+\sqrt{(k+1)(k+2)(k+3)(k+4)} \varphi_{k+4}\right), \\
& \frac{d^{2}}{d x^{2}} \varphi_{k}=\frac{1}{2}\left(\sqrt{k(k-1)} \varphi_{k-2}-(2 k+1) \varphi_{k}+\sqrt{(k+1)(k+2)} \varphi_{k+2}\right) \text {. }
\end{aligned}
$$

We have used the following convention : when any integer becomes negative then we replace it by 0 . 
The suitable spaces are Sobolev spaces with weight are naturally associated to the harmonic oscillator $H_{\text {osc }}$ because the usual Sobolev spaces are associated with the Laplacian. For each integer $m \geq 0$ we define the space $\mathcal{B}_{m}$ of function $u \in L^{2}(\mathbb{R})$ such that for any pair of integers $k, l$ such that $k+\ell \leq m$ we have $x^{k} \frac{d^{\ell}}{d x^{\ell}} u \in L^{2}(\mathbb{R}) . \mathcal{B}_{m}$ is a Hilbert space with the scalar product:

$$
\langle u, v\rangle_{m}=\sum_{k+\ell \leq m} \int_{\mathbb{R}}\left(\overline{x^{k} \frac{d^{\ell}}{d x^{\ell}}} u\right)\left(x^{k} \frac{d^{\ell}}{d x^{\ell}} v\right) d x .
$$

$\mathcal{B}_{m}$ is equal to the domain of $H_{o s c}^{m / 2}$ and the scalar product is equivalent to :

$$
\langle u, v\rangle_{m}^{\star}=\left\langle H_{o s c}^{m / 2} u, H_{o s c}^{m / 2} v\right\rangle=\left\langle H_{o s c}^{m} u, v\right\rangle .
$$

We deduce the following characterization of $\mathcal{B}_{m}$ with the Hermite coefficient of $u, \alpha_{k}(u):=$ $\left\langle\varphi_{k}, u\right\rangle$.

Proposition A.1. $u \in \mathcal{B}_{m}$ if and only if

$$
\sum_{k \in \mathbb{N}}(2 k+1)^{m}\left|\alpha_{k}\right|^{2}<+\infty
$$

In addition, the scalar product is expressed as the following :

$$
\langle u, v\rangle_{m}^{\star}=\sum_{k \in \mathbb{N}}(2 k+1)^{m} \overline{\alpha_{k}(u)} \alpha_{k}(v) .
$$

The proposition can be summarized by saying that $\mathcal{B}_{m}$ is identical to the domain of the operator $H_{o s c}^{m / 2}$. By complex interpolation we deduce the intermediate spaces $\mathcal{B}_{s}$ for all $s$ positive reals hence by the duality for $s$ negative reals. The arguments are identical to the case of usual Sobolev spaces. For $s<0$ the $\mathcal{B}_{s}$ are the spaces of temperate distribution.

We set $u_{N}=\sum_{0 \leq k \leq N} \alpha_{k}(u) \varphi_{k}$, let $u_{N}=\Pi_{N} u, \Pi_{N}$ be the projections on the vector space $V_{N}$ generated by $\left\{\varphi_{0}, \varphi_{1}, \cdots, \varphi_{N}\right\}$. So we clearly have :

$$
\left\|u-u_{N}\right\|^{2}=\sum_{k>N}\left|\alpha_{k}\right|^{2} \leq \frac{1}{(2 N+1)^{m}} \sum_{k \in \mathbb{N}}(2 k+1)^{m}\left|\alpha_{k}\right|^{2} .
$$

Hence if $u \in \mathcal{B}_{m}$ we have :

$$
\left\|u-u_{N}\right\|^{2} \leq(2 N+1)^{-m}\|u\|_{m}^{\star 2} .
$$

More generally we can estimate the error in the spaces $\mathcal{B}_{s}$ :

$$
\left\|u-u_{N}\right\|_{s}^{\star, 2} \leq(2 N+1)^{s-m}\|u\|_{m}^{\star, 2} .
$$

It may be useful to have such Sobolev inequalities explaining the regularity and decay at infinity of $u \in \mathcal{B}_{s}$ as soon as $s$ is large enough : 
Proposition A.2. Let $m \in \mathbb{N}$. There exists constants $C_{m}>0, C_{s, m}(m<2 s-2)$ such that

$$
\begin{aligned}
& \left|x^{\ell} \frac{d^{k}}{d x^{k}} \varphi_{j}(x)\right| \leq C_{m}(2 j+1)^{(m+1) / 2}, \quad \forall x \in \mathbb{R}, k+\ell \leq m, \\
& \left|x^{\ell} \frac{d^{k}}{d x^{k}} u(x)\right| \leq C_{s, m}\|u\|_{s}, \quad \forall x \in \mathbb{R}, k+\ell \leq m, u \in \mathcal{S}(\mathbb{R}) .
\end{aligned}
$$

In particular if $m$ is known and if $s>m+2$ then all $u$ in $\mathcal{B}_{s}$ are of class $C^{m}$ on $\mathbb{R}$ and verify the inequality (A.6).

Thus we see that the functions $u \in \mathcal{B}_{m}$ are both regular and decreasing to 0 at the infinity more rapidly when $m$ is big (positive).

\section{Appendix B Pseudospectra}

The eigenvalues of Schrödinger pencils are very unstable (see Section 2). As proposed some times ago by Trefethen [37] it is useful to replace the spectra of non-self adjoint operators by something more stable which is called the pseudospectra.

Let $A$ be closed operator in the Hilbert space $\mathcal{H}$ with domain $D(A)$ dense in $\mathcal{H}$. Recall that $D(A)$ is an Hilbert space for the graph norm

$$
\|u\|_{D(A)}=\sqrt{\|u\|_{\mathcal{H}}^{2}+\|A u\|_{\mathcal{H}}^{2}} .
$$

Definition B.1. The complex number $z$ is in resolvent set $\rho(A)$ of $A$ if and only if $A-z \mathbb{I}$ is invertible from $D(A)$ into $\mathcal{H}$ and $(A-z \mathrm{II})^{-1} \in \mathcal{L}(\mathcal{H})$ where $\mathcal{L}(\mathcal{H})$ is the Banach space of linear and continuous maps in $\mathcal{H}$. The spectrum $\sigma(A)$ is defined as $\sigma(A)=\mathbb{C} \backslash \rho(A)$

Definition B.2. Consider $\varepsilon>0$. The $\varepsilon$-spectrum $\sigma_{\varepsilon}(A)$ of $A$ is defined as follows. A complex number $z \in \sigma_{\varepsilon}(A)$ if and only if $z \in \sigma(A)$ or if $\left\|(A-z \mathbb{I})^{-1}\right\|_{\mathcal{L}(\mathcal{H})}>\mathcal{E}^{-1}$. It is convenient to write $\left\|(A-z \mathbb{I})^{-1}\right\|_{\mathcal{L}(\mathcal{H})}=\infty$ if $z \in \sigma(A)$ and denote $A-z=A-z \mathbb{I}$.

There are several equivalent definitions of $\sigma_{\varepsilon}(A)$ for details see the introduction of the book [38]. The following characterization is useful for numerical computations. Assume that $\operatorname{dim} \mathcal{H}<+\infty$. Recall that the singular values for $A \in \mathcal{L}(\mathcal{H})$ are the eigenvalues of the non negative matrix $\sqrt{A^{*} A}:=|A|$. Denote $s(A)=\sigma(|A|)$. We have :

Proposition B.1. For any matrix $A, z \in \sigma_{\mathcal{\varepsilon}}(A)$ if and only if $s_{\min }(A-z)<\varepsilon$, where we have denoted $s_{\min }(A):=\min [s(A)]$.

Proof. It is known that $\|A\|=s_{\max }(A)$ for every $A \in \mathcal{L}(\mathcal{H})$. But $A A^{*}$ and $A^{*} A$ have the same non zero eigenvalues, so if $A$ is invertible we have we have $\left\|A^{-1}\right\|=\frac{1}{s_{\min }(A)}$ and the proposition follows. 


\section{References}

[1] Aboud F. Ph.D Thesis, Problèmes aux valeurs propres non-linéaires. University of Nantes, May 2009. https:/ / tel.archives-ouvertes.fr/tel-00410455.

[2] Aboud F, Robert D. Asymptotic expansion for nonlinear eigenvalue problems. J Math Pure Appl, 2010, 93(2): 149-162.

[3] Alam R. On spectral approximation of linear operators. J Mathe Anal Appl, 1998, 226: 229244.

[4] Area I, Dimitrov D K, Godoy E, Ronveaux A. Zeros of Gegenbauer and Hermite polynomials and connection coefficients. Math Comput, 2004, 73(248): 1937-1951.

[5] Asch M, Lebeau G. The spectrum of the damped wave operator for a bounded domain in $\mathbb{R}^{2}$. Exp Math, 2003, 12(2), 227-241.

[6] Bindel D, Hood A. Localization theorems for nonlinear eigenvalue problems. SIAM J Matrix Anal Appl, 2013, 34(4): 1728-1749.

[7] Bramble J H, Osborn J E. Rate of convergence estimates for nonselfadjoint eigenvalue approximations. Math Comput, 1973, 27: 525-549.

[8] Bramble J H, Schatz A H. Rayleigh-Ritz-Galerkin methods for Dirichlet's problem using subspaces without boundary conditions. Comm Pure Appl Math, 1970, 23: 653-675.

[9] Cancès E, Chakir R, Maday Y. Numerical analysis of nonlinear eigenvalue problems. J Sci Comput, 2010, 45(2): 90-117.

[10] Canuto C, Hussaini Y, Quarteroni A, Zang T A. Spectral Methods in Fluid Dynamics. Springer-Verlag, New York, 1988.

[11] Chatelin F. Spectral approximation of linear operators. Classics in applied mathematics 65 Society for Industrial and Applied Mathematics, Philadelphia , 2011.

[12] Chatelin F, Lemordant J. Error bounds in the approximation of eigenvalues of differential and integral operators. J Math Anal Appl, 1978, 62: 257-271.

[13] Ching-Chau Y. Nonlinear eigenvalues and analytic-Hypoellipticity. Memoirs Amer Math Society, 1998, 636.

[14] Christ M. Analytic hypoellipticity, representation of nilpotent groups and non-linear eigenvalue problem. Duke Math J, 1993, 72(3): 595-639.

[15] Christ M. Examples of analytic non-hypoellipticity of $\bar{\partial}_{b}$. Commun PDE, 1994, 19: 911-941.

[16] Christ M. A family of degenerate differential operators. J Geom Anal, 1993, 3: 579-597.

[17] Davies E B. Pseudospectra, the harmonic oscillator and complex resonances. R. Soc Lond Proc Ser , A Math Phys EngSci, 1999, 455: 585-599.

[18] Davies E B, Plum M. Spectral pollution. IMA J Numer Ana, 2004, 24: 417-438.

[19] Effenberger C. Ph.D Thesis, Robust solution methods for nonlinear eigenvalue problems. École Polytechnique Fédérale de Lausanne, Août, 2013.

[20] Gottlieb D, Orszag S A. Numerical analysis of spectral methods: theory and applications. CBMS-NSF Regional Conference Series in Applied Mathematics, SIAM, Philadelphia, 1977.

[21] Grammont L, Higham N J, Tisseur F. A framework for analyzing nonlinear eigenproblems and parametrized linear systems. Linear Alg Appl, 2011, 435: 623-640.

[22] Hansen A C. On the approximation of spectra of linear operators on Hilbert spaces. J Funct Anal, 2008, 254: 2092-2126.

[23] Helffer B. Remarques sur des résultats de G. Métivier sur le nonhypo-analyticité, Séminaire de l'Université de Nantes, exposé, 9: 1978-79.

[24] Higham N, Tisseur F. Structured pseudo spectra for polynomial eigengenvalue problems , with applications. SIAM Matrix Anal Appl, 2001, 23(1): 187-208. 
[25] Higham N, Tisseur F. More on pseudo spectra for polynomial eigenvalue problems and application in control theory. Linear Alg Appl, 2002, 351-352: 435-453.

[26] Krein M G, Langer H. On the mathematical principles in the linear theory of damped oscilations of continua I. Integr Equ Oper Theory 1978, 1/3: 364-399.

[27] Shen J. Efficient spectral-Galerkin method I. Direct solvers for the second and fourth order equations using Legendre polynomials. SIAM J Sci Comput, 1994, 15(6): 1489-1505.

[28] Kolata W, Osborn J. Non-selfadjoint spectral approximation and the finite element method. Springer Verlag. Lect Notes Math, 1979, 701: 115-133.

[29] Llobet X, Appert K, Bondeson A, Vaclavik J. On spectral pollution. Comput Phy Comm, 1990, 59: 199-216.

[30] Markus A S. Introduction to the spectral theory of polynomial operator pencils. Trans Math Monog, 71, AMS, 1988.

[31] Mehrmann V, Voss H. Nonlinear eigenvalue problems : a challenge for modern eigenvalue methods. Mitt Ges Angwandte Math Mechanik 2005, 27: 121-151.

[32] Osborn J E. Spectral approximation for compact operators. Mathematics of Computation 29 (131) (1975), 712-725.

[33] Pham The Lai and D. Robert. Sur un problème aux valeurs propres non linéaires. J. of Math. 36 (1980), 169-186.

[34] Pravda-Starov K. A complete study of the pseudo-spectrum for the rotated harmonic oscillator. J London Math Soc, 2006, 73: 745-761.

[35] Quarteroni A, Sacco R, Saleri F. Numerical Mathematics. Texts in Applied Mathematics, Springer Verlag, 2007.

[36] Tisseur F, Meerbergen K. The quadratic eigenvalue problem. SIAM Review, 2001, 43: 235286.

[37] Trefethen L N. Computation of pseudospectra. Acta Numer, 1999, 8: 247-295.

[38] Trefethen L N, Embree M. Spectra an pseudospectra. Princeton University Press, 2005.

[39] Van Beeumen R, Meerbergen K, Michiels W. A rational Krylov method based on Hermite interpolation for nonlinear eigenvalue problems. SIAM J Sci Comput, 2013, 35(1): A327A350.

[40] Zworski M. Numerical linear algebra and solvability of partial differential equations. Commun Math Phys, 2002, 229: 293-307. 